\title{
DYNAMICS OF WEIGHTED COMPOSITION OPERATORS ON FUNCTION SPACES DEFINED BY LOCAL PROPERTIES
}

\author{
THOMAS KALMES
}

\begin{abstract}
We study topological transitivity/hypercyclicity and topological (weak) mixing for weighted composition operators on locally convex spaces of scalar-valued functions which are defined by local properties. As main application of our general approach we characterize these dynamical properties for weighted composition operators on spaces of ultradifferentiable functions, both of Beurling and Roumieu type, and on spaces of zero solutions of elliptic partial differential equations. Special attention is given to eigenspaces of the Laplace operator and the Cauchy-Riemann operator, respectively. Moreover, we show that our abstract approach unifies existing results which characterize hypercyclicity, resp. topological mixing, of (weighted) composition operators on the space of holomorphic functions on a simply connected domain in the complex plane, on the space of smooth functions on an open subset of $\mathbb{R}^{d}$, as well as results characterizing topological transitiviy of such operators on the space of real analytic functions on an open subset of $\mathbb{R}^{d}$.
\end{abstract}

\section{INTRODUCTION}

Dynamical properties like topological transitivity/hypercyclicity and topological (weak) mixing for weighted composition operators on various function spaces have been investigated by many authors in different settings. Recall, that an operator, i.e. a continuous linear self-map $T$ on a topological vector space $E$, is called topologically transitive, resp. topologically mixing, if for every pair of non-empty, open subsets $U, V$ of $E$ the sets $T^{m}(U)$ and $V$ intersect for some $m \in \mathbb{N}$, resp. for all sufficiently large $m \in \mathbb{N}$, whereas $T$ is called topologically weakly mixing if $T \oplus T$ is topologically transitive on $E \oplus E$. Moreover, $T$ is hypercyclic if there is $x \in E$ such that its orbit under $T$, i.e. the set $\left\{T^{m} x ; m \in \mathbb{N}_{0}\right\}$, is dense in $E$. Clearly, every hypercyclic operator is topologically transitive, and the converse holds for operators on separable, complete, metrizable topological vector spaces by Birkhoff's Transitivity Criterion [GEPe, Theorem 2.19].

The most prominent setting for hypercyclic weighted composition operators is the space of holomorphic functions $\mathscr{H}(X)$ endowed with the compact-open topology on an open subset $X$ of the complex plane. Starting from Birkhoff's result Bi] from which it follows that the translation operators $T_{a}(f)(\cdot):=f(\cdot+a), a \in \mathbb{C} \backslash\{0\}$, on the space of entire functions $\mathscr{H}(\mathbb{C})$ are hypercyclic, many authors have generalized Birkhoff's result into various directions by considering more general (weighted) composition operators $C_{w, \psi}(f):=w \cdot(f \circ \psi)$ for $f \in \mathscr{H}(X)$, where $w \in \mathscr{H}(X)$ and $\psi: X \rightarrow X$ is holomorphic (see e.g. BeMo, GEMo, YoRe, Bes, and the references therein). For holomorphic functions in several variables some partial results on hypercyclicity for special unweighted composition operators have been obtained in [AbZa], $\mathrm{Ber}$, and [LS], and only recently for arbitrary composition

2010 Mathematics Subject Classification. Primary 47A16, 47B33; Secondary 46E10.

Key words and phrases. Hypercyclic operator; Weighted composition operator; Topologically mixing operator; Locally convex spaces of functions; Ultradifferentiable functions; Elliptic differential operator. 
operators (even for holomorphic functions in several variables on a connected Stein manifold) in [Za].

Considering harmonic functions instead of holomorphic functions, in $[\mathrm{Dz}, \mathrm{Ar}$. hypercyclicity of generalized translation operators has been investigated in this context while sufficient conditions for hypercyclicity of such special composition operators on kernels of elliptic partial differential operators have been presented in $\mathrm{CaMu}$, among other things. A sufficient condition for hypercyclicity of general composition operators on arbitrary kernels of partial differential operators was obtained in $\mathrm{KaNi}$.

While for unweighted composition operators on the space of real analytic functions $\mathscr{A}(X)$ on open subsets $X$ of $\mathbb{R}^{d}$ topological transitivity has been investigated in [BoDo, hypercyclicity and topological mixing of weighted composition operators on the space of smooth functions $C^{\infty}(X)$, where again $X \subseteq \mathbb{R}^{d}$ is open, have only very recently been characterized in $[\mathrm{Pr}]$.

For Banach spaces of functions dynamical properties of (weighted) composition operators have also been investigated. There are many results in the context of Banach spaces of holomorphic functions, see e.g. [BoSh, GaMo, MiWo and references therein. Characterizations of hypercyclicity of weighted composition operators on Banach spaces of continuous functions and on $L^{p}(\mu)$-spaces have been obtained in $[\mathrm{Ka}$, among other things.

The aim of the present paper is to present a general approach to topological transitivity and topological (weak) mixing of weighted composition operators on locally convex spaces of scalar valued functions which are defined by local properties. In section 2 we introduce the setting of general locally convex sheaves of functions which gives the appropriate general framework for our objective. This general approach enables us to give an almost characterization of these properties in section 3 (Theorem 3.9 and Theorem 3.11). For many concrete function spaces these almost characterizations can be made into characterizations with only a minimal additional effort. This is shown in section 4 where we not only recover and unify most of the above mentioned results by our general approach but where our abstract setting also permits to improve these results into one direction or the other.

Moreover, as one of the main application of our approach we show in section 5 that topological transitivity and topological weak mixing for weighted composition operators $C_{w, \psi}$ on spaces of ultradifferentiable functions of Beurling type as well as of Roumieu type, respectively, are equivalent and we characterize these properties together with topological mixing in terms of the weight $w$ and the symbol $\psi$ (Theorems 5.2 and 5.3).

As a second main application of our abstract results we show in section 6 that for weighted composition operators on kernels of elliptic partial differential operators in $C^{\infty}(X)$ for open subsets $X$ of $\mathbb{R}^{d}$ which are homeomorphic to $\mathbb{R}^{d}$, hypercyclicity and topological weak mixing are equivalent whenever $|w| \leq 1$, and we again characterize these properties as well as topological mixing in terms of the weight function and the symbol of the operator (Theorem 6.2). We pay special attention to eigenspaces of the Laplace operator (Corollary 6.7) and of the Cauchy-Riemann operator (Corollary 6.8). In the latter case we show that weak mixing, hypercyclicity, and mixing for weighted composition operators coincide whenever $|w| \leq 1$. Additionally, we characterize in terms of the weight and symbol which weighted composition operators are well-defined on the eigenspaces of the Laplace operator and of the Cauchy-Riemann operator (Proposition 6.6). 
Throughout, we use standard notation and terminology from functional analysis. For anything related to functional analysis which is not explained in the text we refer the reader to $\mathrm{MeVO}$. Moreover, we use common notation from the theory of linear partial differential operators. For this we refer the reader to [Hö1]. Finally, for notions and results from dynamics of linear operators which are not explained in the text we refer the reader to BaMa2] and GEPe.

\section{FunCtion SPACES DEFINED By LOCAL PROPERTIES}

In order to deal with weighted composition operators on several function spaces at once we choose the framework of sheaves. Here and in the sequel let $\mathbb{K} \in\{\mathbb{R}, \mathbb{C}\}$.

Definition 2.1. Let $\Omega$ be a locally compact, $\sigma$-compact, non-compact Hausdorff space and $\mathscr{F}$ a sheaf of functions on $\Omega$, i.e.

- For each open subset $X \subseteq \Omega \mathscr{F}(X)$ is a vector space of $\mathbb{K}$-valued functions and if $Y \subseteq \Omega$ is another open set with $Y \subseteq X$ the restriction mapping

$$
r_{X}^{Y}: \mathscr{F}(X) \rightarrow \mathscr{F}(Y), f \mapsto f_{\mid Y}
$$

is well-defined.

- (Localization) For every open cover $\left(X_{\iota}\right)_{\iota \in I}$ of an open set $X \subseteq \Omega$ and for each $f, g \in \mathscr{F}(X)$ with $f_{\mid X_{\iota}}=g_{\mid X_{\iota}}(\iota \in I)$ it holds $f=g$.

- (Gluing) For each open cover $\left(X_{\iota}\right)_{\iota \in I}$ of an open set $X \subseteq \Omega$ and for all $\left(f_{\iota}\right)_{\iota} \in \prod_{\iota \in I} \mathscr{F}\left(X_{\iota}\right)$ with $f_{\iota \mid X_{\iota} \cap X_{\kappa}}=f_{\kappa \mid X_{\iota} \cap X_{\kappa}}(\iota, \kappa \in I)$ there is $f \in \mathscr{F}(X)$ with $f_{\mid X_{\iota}}=f_{\iota}(\iota \in I)$.

From these defining properties of a sheaf of functions it follows immediately that for every open subset $X \subseteq \Omega$ and each open, relatively compact exhaustion $\left(X_{n}\right)_{n \in \mathbb{N}_{0}}$ of $X$ the spaces $\mathscr{F}(X)$ and the projective limit $\operatorname{proj}_{\leftarrow n}\left(\mathscr{F}\left(X_{n}\right), r_{X_{n+1}}^{X_{n}}\right)$, i.e. the subspace

$$
\left\{\left(f_{n}\right)_{n \in \mathbb{N}_{0}} \in \prod_{n \in \mathbb{N}_{0}} \mathscr{F}\left(X_{n}\right) ; \forall n \in \mathbb{N}_{0}: f_{n}=r_{X_{n+1}}^{X_{n}}\left(f_{n+1}\right)\right\}
$$

of $\prod_{n \in \mathbb{N}_{0}} \mathscr{F}\left(X_{n}\right)$ are algebraically isomorphic via the mapping

$$
\mathscr{F}(X) \rightarrow \operatorname{proj}_{\leftarrow n}\left(\mathscr{F}\left(X_{n}\right), r_{X_{n+1}}^{X_{n}}\right), f \mapsto\left(r_{X}^{X_{n}}(f)\right)_{n \in \mathbb{N}_{0}}=\left(f_{\mid X_{n}}\right)_{n \in \mathbb{N}_{0}},
$$

where injectivity follows from the localization property and surjectivity from the gluing property of a sheaf.

In order to be able to apply results from functional analysis, we define the following properties for a sheaf of functions $\mathscr{F}$ on $\Omega$ :

$(\mathscr{F} 1)$ For every open subset $X \subseteq \Omega$ the function space $\mathscr{F}(X)$ is a webbed and ultrabornological Hausdorff locally convex space (which is satisfied, for example, if $\mathscr{F}(X)$ is a Fréchet space). Additionally, we assume that $\mathscr{F}(X) \subseteq C(X)$ for every open $X \subseteq \Omega$ and that for each $x \in X$ the point evaluation $\delta_{x}$ in $x$ is a continuous linear functional on $\mathscr{F}(X)$.

It thus follows that for open $X, Y \subseteq \Omega$ with $Y \subseteq X$ the restriction map $r_{X}^{Y}$ has closed graph, hence is continuous by De Wilde's Closed Graph Theorem (see e.g. [MeVo, Theorem 24.31]).

Moreover, for every open, relatively compact exhaustion $\left(X_{n}\right)_{n \in \mathbb{N}_{0}}$ of $X$ we assume that the above mentioned algebraic isomorphism between $\mathscr{F}(X)$ and $\operatorname{proj}_{\leftarrow n}\left(\mathscr{F}\left(X_{n}\right), r_{X_{n+1}}^{X_{n}}\right)$ is even a topological isomorphism.

$(\mathscr{F} 2)$ For every compact $K \subseteq \Omega$ there is $f_{K} \in \mathscr{F}(\Omega)$ such that $f_{K}(x) \neq 0$ for each $x \in K$.

$(\mathscr{F} 3)$ For every pair of distinct points $x, y$ in $\Omega$ there is $f \in \mathscr{F}(\Omega)$ with $f(x)=0$ and $f(y)=1$. 


\section{Remark 2.2.}

i) For a sheaf $\mathscr{F}$ on $\Omega$ with the property that $\mathscr{F}(X)$ is a locally convex space and $r_{X}^{Y}$ is continuous for each open $Y \subseteq X \subseteq \Omega$ (by definition, this means that $\mathscr{F}$ is a locally convex sheaf on $\Omega$ ) it follows immediately that for any open, relatively compact exhaustion $\left(X_{n}\right)_{n \in \mathbb{N}}$ of $X$ the canonical isomorphism between $\mathscr{F}(X)$ and $\operatorname{proj}_{\leftarrow n}\left(\mathscr{F}\left(X_{n}\right), r_{X_{n+1}}^{X_{n}}\right)$ is continuous. Therefore, if $\mathscr{F}$ is a locally convex sheaf of continuous functions such that $\mathscr{F}(X)$ is a Fréchet space for each open $X \subseteq \Omega$ on which $\delta_{x}$ is a continuous linear functional for every $x \in X$, it follows from the Open Mapping Theorem and the fact that Fréchet spaces are ultrabornological (see e.g. MeVo, Re-

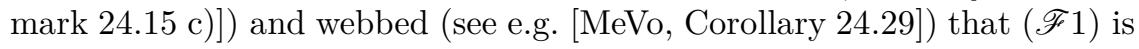
satisfied.

ii) For a sheaf $\mathscr{F}$ on $\Omega$ satisfying $(\mathscr{F} 1)$ it follows from $\delta_{x} \in \mathscr{F}(X)^{\prime}$ for each $x \in X$ that the inclusion mapping

$$
\mathscr{F}(X) \hookrightarrow C(X), f \mapsto f
$$

has closed graph - where we equip $C(X)$ as usual with the compact-open topology. Since $\mathscr{F}(X)$ is supposed to be ultrabornological it follows from De Wilde's Closed Graph Theorem that this inclusion is continuous, i.e. the topology carried by $\mathscr{F}(X)$ is finer than the compact-open topology.

iii) If $\mathscr{F}$ contains constant functions property $(\mathscr{F} 3)$ means precisely that $\mathscr{F}(\Omega)$ separates points.

\section{Example 2.3.}

i) For a $\sigma$-compact, locally compact, non-compact Hausdorff space $\Omega$ the sheaf $C$ of continuous functions satisfies $(\mathscr{F} 1)-(\mathscr{F} 3)$, i.e. for an open subset $X \subseteq$ $\Omega$ let $C(X)$ be the space of $\mathbb{K}$-valued continuous functions on $X$ equipped with the compact-open topology, that is, the locally convex topology defined by the family of seminorms $\left\{\|\cdot\|_{K} ; K \subset X\right.$ compact $\}$, where for a compact subset $K \subset X$

$$
\forall f \in C(X):\|f\|_{K}:=\sup _{x \in K}|f(x)| .
$$

Recall that locally compact spaces are completely regular (see e.g. [En, Theorem 3.3.1]) so that ( $\mathscr{F} 3)$ is indeed satisfied.

ii) For $\Omega=\mathbb{R}^{d}$ and $r \in \mathbb{N}_{0} \cup\{\infty\}$ we denote by $C^{r}$ the sheaf of $r$-times continuously differentiable functions, i.e. for every open $X \subseteq \mathbb{R}^{d}$ let $C^{r}(X)$ be the space of $\mathbb{K}$-valued functions which are $r$-times continuously differentiable. We equip $C^{r}(X)$ with the topology of local uniform convergence of all partial derivatives up to order $r$, i.e. the locally convex topology defined by the family of seminorms $\left\{\|\cdot\|_{l, K} ; l \in \mathbb{N}_{0}, l<r+1, K \subset X\right.$ compact $\}$, where for $l<r+1$ and $K \subset X$ compact

$$
\forall f \in C^{r}(X):\|f\|_{l, K}:=\sup _{|\alpha| \leq l} \sup _{x \in K}\left|\partial^{\alpha} f(x)\right| .
$$

This makes $C^{r}(X)$ a separable Fréchet space and the sheaf $C^{r}$ on $\mathbb{R}^{d}$ is easily seen to satisfy $(\mathscr{F} 1)-(\mathscr{F} 3)$.

iii) For $\Omega=\mathbb{C}$ let $\mathscr{H}$ be the sheaf of holomorphic functions, i.e. for $X \subseteq \mathbb{C}$ let $\mathscr{H}(X)$ denote the space of holomorphic functions on $X$ endowed with the compact-open topology. Then $\mathscr{H}(X)$ is a separable Fréchet space and it follows easily that $(\mathscr{F} 1)-(\mathscr{F} 3)$ are satisfied. More generally than this example is $\mathrm{v}$ ).

iv) Let again $\Omega=\mathbb{R}^{d}$ and denote by $\mathscr{A}$ the sheaf of real analytic functions, that is, for open $X \subseteq \mathbb{R}^{d} \mathscr{A}(X)$ is the space of real analytic functions of $X$. 
One apparent way of equipping $\mathscr{A}(X)$ with a locally convex topology is by considering the finest locally convex topology such that all the restriction maps

$$
\mathscr{H}(U) \rightarrow \mathscr{A}(X), f \mapsto f_{\mid X}
$$

are continuous, where $U \subseteq \mathbb{C}^{d}$ is an arbitrary open set for which $X \subseteq U \cap \mathbb{R}^{d}$ and where $\mathscr{H}(U)$ is equipped with the compact-open topology. It is not hard to see that this (inductive) topology is Hausdorff. Another way of endowing $\mathscr{A}(X)$ with a natural locally convex topology is by taking the initial topology with respect to all restriction maps

$$
r_{K}: \mathscr{A}(X) \rightarrow \mathscr{H}(K), f \mapsto f_{\mid K},
$$

where $K \subseteq X$ is an arbitrary compact set and $\mathscr{H}(K)$ denotes the space of germs of holomorphic functions on $K$ equipped with the locally convex inductive limit topology $\mathscr{H}(K)=\operatorname{ind}_{m \in \mathbb{N}} \mathscr{H}^{\infty}\left(U_{m}\right)$, where $\left(U_{m}\right)_{m \in \mathbb{N}}$ is a (decreasing) basis of $\mathbb{C}^{d}$-neighborhoods of $K$ and $\mathscr{H}^{\infty}\left(U_{m}\right)$ denotes the Banach space of bounded holomorphic functions on $U_{m}$ equipped with the supremum norm. Choosing a compact exhaustion $\left(K_{n}\right)_{n \in \mathbb{N}}$ of $X$ it follows that topologized in this way $\mathscr{A}(X)$ equals the (topological) projective limit of the projective sequence $\left(\mathscr{H}\left(K_{n}\right)\right)_{n \in \mathbb{N}}$ with restrictions as linking maps.

Again, it is not hard to see that the first (inductive) topology on $\mathscr{A}(X)$ is coarser than the second (projective) topology. That these two topologies actually coincide is a fundamental result due to Martineau (see [Mar]). Since $\mathscr{H}(U)$ is a Fréchet space, thus ultrabornological, it follows that $\mathscr{A}(X)$ is ultrabornological as the inductive limit of ultrabornological spaces. As an $L B$-space, $\mathscr{H}(K)$ is webbed for every compact $K \subseteq X$ and therefore $\mathscr{A}(X)$ is webbed as the countable projective limit of webbed spaces.

Now let $X \subseteq \mathbb{R}^{d}$ be open and let $\left(X_{n}\right)_{n \in \mathbb{N}_{0}}$ be an open, relatively compact exhaustion of $X$

$$
i: \mathscr{A}(X) \rightarrow \operatorname{proj}_{\leftarrow n}\left(\mathscr{A}\left(X_{n}\right), r_{X_{n+1}}^{X_{n}}\right), f \mapsto\left(r_{X}^{X_{n}}(f)\right)_{n \in \mathbb{N}_{0}}
$$

We show that the continuous bijection $i$ is open. By the projective description of the topology on $\mathscr{A}(X)$ for any zero neighborhood $V$ in $\mathscr{A}(X)$ there is a compact subset $K$ of $X$ and an absolutely convex zero neighborhood $W$ in $\mathscr{H}(K)$ such that $r_{K}^{-1}(W) \subseteq V$. Thus, for every complex neighborhood $U$ of $K$ there is $\delta>0$ with

$$
r_{K}^{-1}\left(\left\{g \in \mathscr{H}(K) ; g \in \mathscr{H}^{\infty}(U),\|g\|_{\infty, U}<\delta\right\}\right) \subseteq V,
$$

where $\|\cdot\|_{\infty, U}$ denotes the supremum norm over $U$. If $m \in \mathbb{N}_{0}$ is chosen such that $K \subseteq X_{m}$ it follows with

$$
\rho_{K}: \mathscr{A}\left(X_{m}\right) \rightarrow \mathscr{H}(K), f \mapsto f_{\mid K}
$$

that

$$
V_{m}:=\rho_{K}^{-1}\left(\left\{g \in \mathscr{H}(K) ; g \in \mathscr{H}^{\infty}(U),\|g\|_{\infty, U}<\delta\right\}\right)
$$

is a zero neighborhood in $\mathscr{A}\left(X_{m}\right)$. Clearly,

$$
\begin{aligned}
V_{m} \cap r_{X}^{X_{m}}(\mathscr{A}(X)) & =r_{X}^{X_{m}}\left(r_{K}^{-1}\left(\left\{g \in \mathscr{H}(K) ; g \in \mathscr{H}^{\infty}(U),\|g\|_{\infty, U}<\delta\right\}\right)\right) \\
& \subseteq r_{X}^{X_{m}}(V) .
\end{aligned}
$$

If we set for $k \in \mathbb{N}_{0}$

$$
\pi_{k}: \operatorname{proj}_{\leftarrow n}\left(\mathscr{A}\left(X_{n}\right), r_{X_{n+1}}^{X_{n}}\right) \rightarrow \mathscr{A}\left(X_{k}\right),\left(f_{n}\right)_{n \in \mathbb{N}_{0}} \mapsto f_{k}
$$

it follows

$$
i(V)=\pi_{m}^{-1}\left(r_{X}^{X_{m}}(V)\right) \supseteq \pi_{m}^{-1}\left(V_{m} \cap r_{X}^{X_{m}}(\mathscr{A}(X))\right)=\pi_{m}^{-1}\left(V_{m}\right)
$$


so that $i(V)$ is a zero neighborhood in $\operatorname{proj}_{\leftarrow n}\left(\mathscr{A}\left(X_{n}\right), r_{X_{n+1}}^{X_{n}}\right)$ which proves that $i$ is open. Thus, the sheaf $\mathscr{A}$ on $\mathbb{R}^{d}$ satisfies $(\mathscr{F} 1)$. Moreover, $(\mathscr{F} 2)$ as well as $(\mathscr{F} 3)$ are obviously satisfied, too.

v) For $\Omega=\mathbb{R}^{d}$ and a complex coefficient polynomial in $d$ variables $P$, i.e. $P \in \mathbb{C}\left[X_{1}, \ldots, X_{d}\right]$ we define for an open $X \subseteq \mathbb{R}^{d}$

$$
C_{P}^{\infty}(X):=\left\{f \in C^{\infty}(X) ; P(\partial) f=0 \text { in } X\right\},
$$

where as usual for $P(\xi)=\sum_{|\alpha| \leq m} a_{\alpha} \xi^{\alpha}$ we set

$$
\forall f \in C^{\infty}(X), x \in X: P(\partial) f(x)=\sum_{|\alpha| \leq m} a_{\alpha} \partial^{\alpha} f(x) .
$$

Obviously, $C_{P}^{\infty}(X)$ is a subspace of $C^{\infty}(X)$ and since $P(\partial)$ is a continuous linear operator on the separable Fréchet space $C^{\infty}(X)$ it follows that $C_{P}^{\infty}(X)$ is a closed subspace of $C^{\infty}(X)$ thus a separable Fréchet space itself when equipped with the relative topology of $C^{\infty}(X)$. It is easily seen that $C_{P}^{\infty}$ is a sheaf on $\mathbb{R}^{d}$ which satisfies $(\mathscr{F} 1)$. In order to see that $(\mathscr{F} 2)$ holds as well, let $\zeta \in \mathbb{C}^{d}$ satisfy $P(\zeta)=0$ - we exclude the rather boring case of a constant polynomial $P$. Then

$$
e_{\zeta}: \mathbb{R}^{d} \rightarrow \mathbb{C}, e_{\zeta}(x)=\exp \left(\sum_{j=1}^{d} \zeta_{j} x_{j}\right)
$$

belongs to $C_{P}^{\infty}\left(\mathbb{R}^{d}\right)$ which shows that $(\mathscr{F} 2)$ is indeed satisfied.

However, $(\mathscr{F} 3)$ need not be satisfied for general (non-constant) $P$ as is seen by taking $d=2$ and $P\left(\xi_{1}, \xi_{2}\right)=\xi_{1}$. Then $C_{P}^{\infty}(X)$ consists obviously of functions which are independ on $x_{1}$ and there is no $f \in C_{P}^{\infty}\left(\mathbb{R}^{2}\right)$ with $f(0,0)=1$ and $f(1,0)=0$. However, by Proposition 6.1 (Fु3) is in particular satisfied by $C_{P}^{\infty}$ whenever $P$ is (hypo)elliptic.

Considering the special cases of $P(\partial)$ being the Cauchy-Riemann operator, resp. the Laplace operator, gives as $C_{P}^{\infty}$ the sheaf of holomorphic functions $\mathscr{H}$ on open subsets of $\mathbb{C}$, resp. the sheaf of harmonic functions on open subsets of $\mathbb{R}^{d}$.

In most of the above examples the considered sheaves are sheaves of $C^{1}$-functions over open subsets of euclidean space. For these kind of sheaves we introduce yet another property.

Definition 2.4. Let $\Omega=\mathbb{R}^{d}$ and let $\mathscr{F}$ be a sheaf satisfying $(\mathscr{F} 1)$. Then we define:

$(\mathscr{F} 4)$ For every open $X \subseteq \mathbb{R}^{d}$ we have $\mathscr{F}(X) \subseteq C^{1}(X)$ and for every $x \in X$, $1 \leq j \leq d$ the distribution of order one

$$
-\partial_{j} \delta_{x}: \mathscr{F}(X) \rightarrow \mathbb{K}, f \mapsto \partial_{j} f(x)
$$

is continuous. Moreover, for each $h \in \mathbb{R}^{d} \backslash\{0\}, \lambda \in \mathbb{K}$, and $x \in X$ the kernel of the continuous linear functional

$$
\mathscr{F}(X) \rightarrow \mathbb{K}, f \mapsto \sum_{j=1}^{d} h_{j} \partial_{j} f(x)-\lambda f(x)
$$

is not all of $\mathscr{F}(X)$.

Property $(\mathscr{F} 4)$ implies that for every direction $h \in \mathbb{R}^{d} \backslash\{0\}$ and any $x \in X$ the directional derivative in direction $h$ evaluated at $x$ does not coincide with a multiple of $\delta_{x}$. Clearly, $(\mathscr{F} 4)$ is satisfied by Examples [2.3 ii) for $r \geq 1$, iii), and iv). For hypoelliptic polynomials $P$ the sheaf $C_{P}^{\infty}$ satisfies $(\mathscr{F} 4)$ by Proposition 6.1, 
General assumption. Let $\mathscr{F}$ be a sheaf on $\Omega$ satisfying $(\mathscr{F} 1), X \subseteq \Omega$ open, and let $w: X \rightarrow \mathbb{K}$ as well as $\psi: X \rightarrow X$ be continuous. $w$ will be called a weight and $\psi$ a symbol. We assume that the weighted composition operator

$$
C_{w, \psi}: \mathscr{F}(X) \rightarrow \mathscr{F}(X), f \mapsto w \cdot(f \circ \psi)
$$

is well-defined. For every $x \in X$ we have by hypothesis that $\delta_{x} \in \mathscr{F}(X)^{\prime}$ and it follows easily from the Hahn-Banach Theorem, that the linear span of $\left\{\delta_{x} ; x \in X\right\}$ is weak*-dense in $\mathscr{F}(X)^{\prime}$. Since $\mathscr{F}(X)$ is Hausdorff, it follows herefrom that $C_{w, \psi}$ has closed graph. By $(\mathscr{F} 1)$ we conclude from De Wilde's Closed Graph Theorem MeVo, Theorem 24.31] that $C_{w, \psi}$ is continuous.

\section{Dynamical properties of Weighted COMposition operators}

In this section we give necessary and sufficient conditions for a weighted composition operator defined on a local space of functions to be topologically transitive and topologically (weakly) mixing, respectively. We will see that in many concrete cases these necessary and sufficient conditions coincide thus they yield a characterization of said properties.

Definition 3.1. Let $E$ be a locally convex space and $T$ a continuous linear operator on $E$.

i) $T$ is called (topologically) transitive if for any pair of non-empty, open subsets $U, V$ of $E$ there is $m \in \mathbb{N}$ such that $T^{m}(U) \cap V \neq \emptyset$.

ii) $T$ is called (topologically) weakly mixing if $T \oplus T$ is transitive on $E \oplus E$, i.e. if for every choice of non-empty, open subsets $U_{j}, V_{j}$ of $E, j=1,2$, there is $m \in \mathbb{N}$ such that $T^{m}\left(U_{j}\right) \cap V_{j} \neq \emptyset$.

iii) $T$ is called (topologically) mixing if for every pair $U, V$ of non-empty, open subsets of $E$ there is $M \in \mathbb{N}$ such that $T^{m}(U) \cap V \neq \emptyset$ for every $m \geq M$.

iv) $T$ is called hypercyclic if there is $x \in E$ whose orbit under $T$, i.e. $\operatorname{orb}(T, x):=$ $\left\{T^{m} x ; m \in \mathbb{N}_{0}\right\}$ is dense in $E$.

Remark 3.2. $\quad$ i) Clearly, $E$ has to be separable in order to support a hypercyclic operator. By Birkhoff's Transitivity Criterion GEPe, Theorem 2.19] a continuous linear operator on a separable Fréchet space is transitive if and only if it is hypercyclic.

ii) Obviously, every mixing operator is weakly mixing and every weakly mixing operator is transitive. In general, the reverse implications are not true. While it is not too complicated to give an example of a weakly mixing operator which is not mixing (see e.g. GEPe, Remark 4.10]) it is highly intricate to construct a hypercyclic operator (on a Banach space) which fails to be weakly mixing. Such an operator was constructed by De la Rosa and Read DR (see also BaMa] who thereby solved a problem posed by Herrero $\mathrm{He}$ which remained open for more than fifteen years.

iii) It follows immediately from the definition that every transitive operator has dense image.

We first give necessary conditions for a weighted composition operator defined on a local space of functions to be transitive. This result is inspired by $\mathrm{Pr}$, Lemma 3.1]. Before we state the condition we recall a definition for symbols.

Definition 3.3. Let $X \subseteq \Omega$ be open and $\psi: X \rightarrow X$ be continuous.

i) $\psi$ is called run-away if for each compact subset $K$ of $X$ there is $m \in \mathbb{N}$ such that $\psi^{m}(K) \cap K=\emptyset$, where $\psi^{m}$ denotes the $m$-fold iterate of $\psi$, i.e. $\psi^{m}=\psi \circ \ldots \circ \psi$ with $m$ composition factors.

ii) $\psi$ is called strong run-away if for each compact subset $K$ of $X$ there is $M \in \mathbb{N}$ such that $\psi^{m}(K) \cap K=\emptyset$ whenever $m \geq M$. 
Clearly, strong run-away implies run-away. The converse is true for continuous and injective $\psi: \mathbb{R} \rightarrow \mathbb{R}$, as has been shown in $[\mathrm{Pr}$, Lemma 4.1]. Moreover, for a holomorphic $\psi: X \rightarrow X$ on a simply connected domain $X \subsetneq \mathbb{C}$ run-away and strong run-away are the same, due to the Riemann Mapping Theorem combined with the Denjoy-Wolff Iteration Theorem (see e.g. Sh, Chapter 5]) and in fact these properties are equivalent to $\psi$ having no fixed point. To the author's best knowledge, there is no example of a continuous $\psi: X \rightarrow X$ on an open subset $X$ of a locally compact, $\sigma$-compact, non-compact Hausdorff space $\Omega$ which is run-away but not strong run-away.

Proposition 3.4. Let $\mathscr{F}$ be a sheaf on $\Omega$ satisfying $(\mathscr{F} 1)-(\mathscr{F} 3)$. Let $X \subseteq \Omega$ be non-empty and open and assume that the weighted composition operator $C_{w, \psi}$ is transitive on $\mathscr{F}(X)$. Then the following hold.

i) $\forall x \in X: w(x) \neq 0$.

ii) $\psi$ is injective.

iii) $\psi$ has no fixed points.

iv) $\forall x \in X: \overline{\left\{\psi^{m}(x) ; m \in \mathbb{N}_{0}\right\}}$ is not a compact subset of $X$, where the closure is taken in $X$.

v) $\psi$ is run-away under any of the following additional assumptions: v-1) $\forall x \in X:|w(x)| \leq 1$.

$\mathrm{v}-2) C_{w, \psi}$ is weakly mixing.

$\mathrm{v}-3) \mathscr{F}(\Omega)$ is dense in $C(\Omega)$ with respect to the compact-open topology.

Additionally, if $\mathscr{F}$ satisfies $(\mathscr{F} 4)$ then we also have

vi) $\forall x \in X: \operatorname{det} J \psi(x) \neq 0$, where $J \psi(x)$ denotes the Jacobian of $\psi$ in $x$.

Proof. In order to prove i), assume that $w\left(x_{0}\right)=0$ for some $x_{0} \in X$. Then the image of $C_{w, \psi}$ is contained in the kernel of $\delta_{x_{0}}$ which is a closed subspace of $\mathscr{F}(X)$ due to $(\mathscr{F} 1)$. By $(\mathscr{F} 3)$ this closed subspace is proper, in particular nowhere dense. Hence, the image of $C_{w, \psi}$ is not dense in $\mathscr{F}(X)$ contradicting that $C_{w, \psi}$ is transitive, so that i) follows.

Next, we assume that $\psi(x)=\psi(y)$ for $x, y \in X, x \neq y$. Because $w(y) \neq 0$ by i) it follows

$$
\operatorname{im} C_{w, \psi} \subseteq \operatorname{kern}\left(\delta_{x}-\frac{w(x)}{w(y)} \delta_{y}\right)
$$

Since $w(x) \neq 0$ by i) it follows from $(\mathscr{F} 1)$ and $(\mathscr{F} 3)$ that kern $\left(\delta_{x}-\frac{w(x)}{w(y)} \delta_{y}\right)$ is a closed, proper subspace of $\mathscr{F}(X)$. Since the image of $C_{w, \psi}$ is dense in $\mathscr{F}(X)$ we obtain a contradiction as in the proof of $i$ ), so that ii) follows.

To prove iii) we denote for $\alpha \in \mathbb{K}$ and $r>0$ the open ball about $\alpha$ with radius $r$ as $B(\alpha, r)$ and the corresponding closed ball by $B[\alpha, r]$. Assume there is $x_{0} \in X$ with $\psi\left(x_{0}\right)=x_{0}$. In case of $\left|w\left(x_{0}\right)\right| \leq 1$ we have

$$
\forall f \in \delta_{x_{0}}^{-1}(B(0,1)), n \in \mathbb{N}_{0}:\left|C_{w, \psi}^{n}(f)\left(x_{0}\right)\right| \leq 1
$$

and thus

$$
\forall n \in \mathbb{N}_{0}: C_{w, \psi}^{n}\left(\delta_{x_{0}}^{-1}(B(0,1))\right) \cap \delta_{x_{0}}^{-1}(B(2,1))=\emptyset .
$$

But since kern $\delta_{x_{0}} \neq \mathscr{F}(X)$ by $(\mathscr{F} 3)$ we have im $\delta_{x_{0}}=\mathbb{K}$ so $\delta_{x_{0}}^{-1}(B(2,1))$ is a nonempty, open (by $(\mathscr{F} 1)$ ) subset of $\mathscr{F}(X)$, as is $\delta_{x_{0}}^{-1}(B(0,1))$. Hence, (1) contradicts the transitivity of $C_{w, \psi}$. In case of $\left|w\left(x_{0}\right)\right|>1$ we have

$$
\forall f \in \delta_{x_{0}}^{-1}(\mathbb{K} \backslash B[0,1]), n \in \mathbb{N}:\left|C_{w, \psi}^{n}(f)\left(x_{0}\right)\right|>1
$$

implying

$$
\forall n \in \mathbb{N}_{0}: C_{w, \psi}^{n}\left(\delta_{x_{0}}^{-1}(\mathbb{K} \backslash B[0,1])\right) \cap \delta_{x_{0}}^{-1}(B(0,1))=\emptyset
$$

which again contradicts the transitivity of $C_{w, \psi}$. Thus, iii) is proved. 
In order to prove iv), assume that for some $x_{0} \in X$ the set

$$
K:=\overline{\left\{\psi^{m}\left(x_{0}\right) ; m \in \mathbb{N}_{0}\right\}}
$$

is compact. By i), there are $a, b>0$ such that

$$
\forall x \in K: a \leq|w(x)| \leq b .
$$

Fix $f_{K}$ according to $(\mathscr{F} 2)$ and set

$$
\alpha:=\inf _{x \in K}\left|f_{K}(x)\right| \text { and } \beta:=\sup _{x \in K}\left|f_{K}(x)\right| .
$$

Then $\alpha>0$ and $\beta<\infty$ and the set

$$
U:=\left\{f \in \mathscr{F}(X) ; \forall x \in K: \frac{\alpha}{2}<|f(x)|<2 \beta\right\}
$$

obviously contains $f_{K}$ and is open with respect to the compact-open topology. Thus, $U$ is an open neighborhood of $f_{K}$ in $\mathscr{F}(X)$. A straightforward calculation gives

$$
\forall f \in U, m \in \mathbb{N}_{0}:\left|\frac{C_{w, \psi}^{m}(f)\left(\psi\left(x_{0}\right)\right)}{C_{w, \psi}^{m}(f)\left(x_{0}\right)}\right| \leq \frac{4 b \beta}{a \alpha} .
$$

By $(\mathscr{F} 1)$ it follows that

$$
V:=\left\{f \in \mathscr{F}(X) ;\left|\delta_{\psi\left(x_{0}\right)}(f)\right|>\frac{4 b \beta}{a \alpha}\left|\delta_{x_{0}}(f)\right|\right\}
$$

is an open subset of $\mathscr{F}(X)$ and since $\psi\left(x_{0}\right) \neq x_{0}$ by iii) it follows from $(\mathscr{F} 3)$ that $V \neq \emptyset$. From (2) we obtain

$$
\forall m \in \mathbb{N}_{0}: C_{w, \psi}^{m}(U) \cap V=\emptyset
$$

which contradicts the transitivity of $C_{w, \psi}$. Thus, iv) is proved.

We continue with the proof of $\mathrm{v}$ ) and argue again by contradiction. Assume there is a compact subset $K$ of $X$ with

$$
\forall m \in \mathbb{N} \exists x_{m} \in K: \psi^{m}\left(x_{m}\right) \in K .
$$

Due to $(\mathscr{F} 2)$ there is $f_{K} \in \mathscr{F}(X)$ with $f_{K}(x) \neq 0$ for every $x \in K$. We set

$$
\alpha:=\inf _{y \in K}\left|f_{K}(y)\right|>0, \beta:=\sup _{y \in K}\left|f_{K}(y)\right|<\infty .
$$

We first assume that additionally $|w(x)| \leq 1$ holds for every $x \in X$. We define

$$
U_{1}:=\left\{g \in \mathscr{F}(X) ; \forall x \in K:|g(x)|<\frac{\alpha}{2}\right\}
$$

and

$$
V_{1}:=\left\{g \in \mathscr{F}(X) ; \forall x \in K:\left|f_{K}(x)-g(x)\right|<\frac{\alpha}{2}\right\}
$$

which are open with respect to the compact-open topology and therefore, by $(\mathscr{F} 1)$, open subsets of $\mathscr{F}(X)$. Obviously, $f_{K} \in V_{1}$ and $0 \in U_{1}$. For every $m \in \mathbb{N}$ and each $g \in U_{1}$ it follows from $|w| \leq 1$ and $x_{m}, \psi^{m}\left(x_{m}\right) \in K$

$$
\begin{aligned}
\max _{x \in K}\left|f_{K}(x)-C_{w, \psi}^{m}(g)(x)\right| & \geq\left|f_{K}\left(x_{m}\right)-\prod_{j=0}^{m-1} w\left(\psi^{j}\left(x_{m}\right)\right) g\left(\psi^{m}\left(x_{m}\right)\right)\right| \\
& \geq \alpha-\left|g\left(\psi^{m}\left(x_{m}\right)\right)\right|>\frac{\alpha}{2}
\end{aligned}
$$

so that $C_{w, \psi}^{m}\left(U_{1}\right) \cap V_{1}=\emptyset$ which gives the desired contradiction to the transitivity of $C_{w, \psi}$.

Next we assume that $C_{w, \psi}$ is even weakly mixing. We define

$$
U_{2}:=\left\{g \in \mathscr{F}(X) ; \forall x \in K: \frac{\alpha}{2}<|g(x)|<2 \beta\right\}
$$


and

$$
V_{2}:=\left\{g \in \mathscr{F}(X) ; \sup _{x \in K}|g(x)|<\frac{\alpha^{2}}{8 \beta}\right\}
$$

which are open with respect to the compact-open topology and thus open subsets of $\mathscr{F}(X)$ by $(\mathscr{F} 1)$. Because $0 \in V_{2}$ and $f_{K} \in U_{2}$ and since $C_{w, \psi}$ is weakly mixing there is $m \in \mathbb{N}$ such that

$$
C_{w, \psi}^{m}\left(U_{2}\right) \cap U_{2} \neq \emptyset \text { and } C_{w, \psi}^{m}\left(U_{2}\right) \cap V_{2} \neq \emptyset .
$$

Pick $f \in U_{2}$ with $C_{w, \psi}^{m}(f) \in U_{2}$. By $\psi^{m}\left(x_{m}\right) \in K$ we have $\left|f\left(\psi^{m}\left(x_{m}\right)\right)\right|<2 \beta$ so that because of $x_{m} \in K$

$$
\begin{aligned}
\frac{\alpha}{2} & <\left|C_{w, \psi}^{m}(f)\left(x_{m}\right)\right|=\left|\prod_{j=0}^{m-1} w\left(\psi^{j}\left(x_{m}\right)\right) f\left(\psi^{m}\left(x_{m}\right)\right)\right| \\
& \leq\left|\prod_{j=0}^{m-1} w\left(\psi^{j}\left(x_{m}\right)\right)\right| 2 \beta .
\end{aligned}
$$

On the other hand, with $g \in U_{2}$ such that $C_{w, \psi}^{m}(g) \in V_{2}$ it follows from $\psi^{m}\left(x_{m}\right) \in K$ and thus $\left|g\left(\psi^{m}\left(x_{m}\right)\right)\right|>\alpha / 2$ with $x_{m} \in K$

$$
\frac{\alpha^{2}}{8 \beta}>\left|C_{w, \psi}^{m} g\left(x_{m}\right)\right|=\left|\prod_{j=0}^{m-1} w\left(\psi^{j}\left(x_{m}\right)\right) g\left(\psi^{m}\left(x_{m}\right)\right)\right|>\frac{\alpha}{2}\left|\prod_{j=0}^{m-1} w\left(\psi^{j}\left(x_{m}\right)\right)\right|
$$

which contradicts (3).

In order to finish the proof of v), we next assume that $\mathscr{F}(\Omega)$ is dense in $C(\Omega)$. Because of i) there are $a, b>0$ such that

$$
\forall x \in K: a \leq|w(x)| \leq b .
$$

By $(\mathscr{F} 2)$ there is $\tilde{f}_{K} \in \mathscr{F}(X)$ such that $\tilde{f}_{K}(x) \neq 0$ for every $x \in K \cup \psi(K)$ so that

$$
\tilde{\alpha}:=\inf _{x \in K \cup \psi(K)}\left|\tilde{f}_{K}(x)\right|>0 \text { and } \tilde{\beta}:=\sup _{x \in K \cup \psi(K)}\left|\tilde{f}_{K}(x)\right|<\infty \text {. }
$$

We define

$$
U_{3}:=\left\{g \in \mathscr{F}(X) ; \forall x \in K \cup \psi(K): \frac{\tilde{\alpha}}{2}<|g(x)|<2 \tilde{\beta}\right\}
$$

which is an open neighborhood of $\tilde{f}_{K}$ in $\mathscr{F}(X)$ satisfying

$$
\forall g \in U_{3}, m \in \mathbb{N}_{0}:\left|\frac{C_{w, \psi}^{m}(g)\left(\psi\left(x_{m}\right)\right)}{C_{w, \psi}^{m}(g)\left(x_{m}\right)}\right| \leq \frac{4 b \tilde{\beta}}{a \tilde{\alpha}},
$$

that is

$$
\forall g \in U_{3}, m \in \mathbb{N}_{0}:\left|\delta_{\psi\left(x_{m}\right)}\left(C_{w, \psi}^{m}(g)\right)\right| \leq \frac{4 b \tilde{\beta}}{a \tilde{\alpha}}\left|\delta_{x_{m}}\left(C_{w, \psi}^{m}(g)\right)\right| .
$$

In particular

$$
\forall m \in \mathbb{N}_{0}: C_{w, \psi}^{m}\left(U_{3}\right) \cap\left(V_{3} \cap \mathscr{F}(X)\right)=\emptyset,
$$

where

$$
V_{3}:=\left\{g \in C(X) ; \forall x \in K:\left|\delta_{\psi(x)}(g)\right|>\frac{4 b \tilde{\beta}}{a \tilde{\alpha}}\left|\delta_{x}(g)\right|\right\} .
$$

Once we have shown that $V_{3} \cap \mathscr{F}(X)$ is a non-empty open subset of $\mathscr{F}(X)$ this will yield the desired contradiction to the transitivity of $C_{w, \psi}$.

A straightforward calculation shows that for $g, h \in C(X)$ and $x \in K$ we have

$$
\left|\delta_{\psi(x)}(h)\right|-\frac{4 b \tilde{\beta}}{a \tilde{\alpha}}\left|\delta_{x}(h)\right| \geq\left|\delta_{\psi(x)}(g)\right|-\frac{4 b \tilde{\beta}}{a \tilde{\alpha}}\left|\delta_{x}(g)\right|-\frac{8 b \tilde{\beta}}{a \tilde{\alpha}} \sup _{y \in K \cup \psi(K)}|g(y)-h(y)|
$$


which shows that $V_{3}$ is an open subset of $C(X)$ with respect to the compact-open topology, thus $V_{3} \cap \mathscr{F}(X)$ is open in $\mathscr{F}(X)$ by $(\mathscr{F} 1)$. Since $\mathscr{F}(\Omega)$ is dense in $C(\Omega)$ and $\left\{f_{\mid X} ; f \in C(\Omega)\right\}$ is dense in $C(X)$ by an application of Urysohn's Lemma En, Theorem 1.5.11], it is enough to show that $V_{3}$ is not empty in order to prove $V_{3} \cap \mathscr{F}(X) \neq \emptyset$.

In order to show that $V_{3} \neq \emptyset$ we use a clever construction from $\mathrm{Pr}$, Lemma 3.2]. Let $Y \subseteq X$ be an open, relatively compact neighborhood of $K$. By Urysohn's Lemma there is $h \in C(X)$ such that $h_{\mid K}=0, h_{\mid X \backslash Y}=1$ and $0 \leq h \leq 1$. The series

$$
f:=\sum_{m=0}^{\infty}\left(\frac{a \alpha}{4 b \beta+a \alpha}\right)^{m} h \circ \psi^{m}
$$

converges uniformly on $X$, in particular $f \in C(X)$ and because of iv) it follows that $f(x)>0$ for all $x \in X$. Moreover, for each $x \in K$ we have

$$
\left|\delta_{\psi(x)}(f)\right|=f(\psi(x))=\frac{4 b \beta+a \alpha}{a \alpha} f(x)>\frac{4 b \beta}{a \alpha}\left|\delta_{x}(f)\right|,
$$

i.e. $f \in V_{3}$. Thus, v) is proved.

Finally, let $\mathscr{F}$ satisfy $(\mathscr{F} 4)$ in addition to $(\mathscr{F} 1)-(\mathscr{F} 3)$. Assuming the existence of $x_{0} \in X$ with $\operatorname{det} J \psi\left(x_{0}\right)=0$ there is $h \in \mathbb{R}^{d} \backslash\{0\}$ in the kernel of $J \psi\left(x_{0}\right)$. By an easy calculation we have

$$
\forall f \in \mathscr{F}(X):\left\langle\nabla C_{w, \psi}(f)\left(x_{0}\right), h\right\rangle=C_{w, \psi}(f)\left(x_{0}\right) \frac{1}{w\left(x_{0}\right)}\left\langle\nabla w\left(x_{0}\right), h\right\rangle,
$$

showing

$$
\operatorname{im} C_{w, \psi} \subseteq \operatorname{kern}\left(f \mapsto\left\langle\nabla f\left(x_{0}\right), h\right\rangle-\frac{1}{w\left(x_{0}\right)}\left\langle\nabla w\left(x_{0}\right), h\right\rangle \delta_{x_{0}}(f)\right) .
$$

By $(\mathscr{F} 4)$ the previously mentioned kernel is a closed, proper subspace of $\mathscr{F}(X)$ which contradicts that the image of $C_{w, \psi}$ is dense in $C_{w, \psi}$.

Definition 3.5. Let $\mathscr{F}$ be a sheaf on $\Omega$ satisfying $(\mathscr{F} 1)$ and $X \subseteq \Omega$ be open such that the weighted composition operator $C_{w, \psi}$ is well-defined on $\mathscr{F}(X) . C_{w, \psi}$ is said to act locally on $\mathscr{F}(X)$ if for every open subset $Y$ of $X$

$$
C_{w, \psi, Y}: \mathscr{F}(Y) \rightarrow \mathscr{F}\left(\psi^{-1}(Y)\right), f \mapsto w \cdot(f \circ \psi),
$$

i.e. the weighted composition operator $C_{w, \psi}$ (formally) applied to functions defined only on $Y$, is well-defined.

Remark 3.6. Clearly, under hypothesis $(\mathscr{F} 1)$, for every open subset $Y$ of $\Omega$ and any $f \in \mathscr{F}(Y)$ the function

$$
\psi^{-1}(Y) \rightarrow \mathbb{K}, y \mapsto w(y) f(\psi(y))
$$

is a well-defined continuous function.

If $C_{w, \psi}$ operates locally on $\mathscr{F}(X)$ it follows from $(\mathscr{F} 1)$ and De Wildes's Closed Graph Theorem that $C_{w, \psi, Y}$ is continuous from $\mathscr{F}(Y)$ to $\mathscr{F}\left(\psi^{-1}(Y)\right)$ for any open subset $Y$ of $X$. It follows immediately from the definition of the restriction maps $r_{X}^{Y}$ etc. and $C_{w, \psi, Y}$ that

$$
C_{w, \psi, Y} \circ r_{X}^{Y}=r_{X}^{\psi^{-1}(Y)} \circ C_{w, \psi},
$$

or more generally

$$
\forall m \in \mathbb{N}: C_{w, \psi,\left(\psi^{m-1}\right)^{-1}(Y)} \circ \ldots \circ C_{w, \psi, Y} \circ r_{X}^{Y}=r_{X}^{\left(\psi^{m}\right)^{-1}(Y)} \circ C_{w, \psi}^{m}
$$

for every open $Y \subseteq X$. 
Proposition 3.7. Let $\mathscr{F}$ be a sheaf on $\Omega$ satisfying $(\mathscr{F} 1), X \subseteq \Omega$ be open such that $C_{w, \psi}$ acts locally on $\mathscr{F}(X)$. Let $\left(X_{n}\right)_{n \in \mathbb{N}}$ be a relatively compact, open exhaustion of $X$. Assume that the following conditions are satisfied

a) For every open, relatively compact subset $Y$ of $X$ and any $m \in \mathbb{N}_{0}$

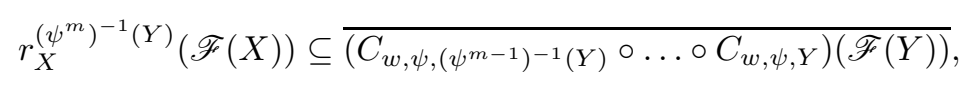

where the closure is taken in $\mathscr{F}\left(\left(\psi^{m}\right)^{-1}(Y)\right)$.

b) There are $m, n \in \mathbb{N}$ with the properties

b1) $\psi^{m}\left(X_{n}\right)$ is an open subset of $X$.

b2) $X_{n} \cap \psi^{m}\left(X_{n}\right)=\emptyset$.

b3) The restriction map $r_{X}^{X_{n} \cup \psi^{m}\left(X_{n}\right)}$ has dense range.

Then, for every $f, g \in \mathscr{F}(X)$ and any absolutely convex zero neighborhood $U_{n}$ in $\mathscr{F}\left(X_{n}\right)$ we have

$$
\emptyset \neq C_{w, \psi}^{m}\left(f+\left(r_{X}^{X_{n}}\right)^{-1}\left(U_{n}\right)\right) \cap\left(g+\left(r_{X}^{X_{n}}\right)^{-1}\left(U_{n}\right)\right)
$$

Proof. Fix $f, g \in \mathscr{F}(X)$ and an absolutely convex zero neighborhood $U_{n}$ in $\mathscr{F}\left(X_{n}\right)$. By b1), $Y:=\psi^{m}\left(X_{n}\right)$ is an open, relatively compact subset of $X$ and $Z:=$ $\left(\psi^{m}\right)^{-1}(Y)$ is an open subset of $X$ with $X_{n} \subseteq Z$ so that $\left(r_{Z}^{X_{n}}\right)^{-1}\left(\frac{1}{2} U_{n}\right)$ is a zero neighborhood in $\mathscr{F}(Z)$. By hypothesis a), there is $\tilde{g} \in \mathscr{F}(Y)$ such that with

$$
T:=C_{w, \psi,\left(\psi^{m-1}\right)^{-1}(Y)^{\circ} \ldots \circ C_{w, \psi, Y}}
$$

(note that $T$ is continuous) we have

$$
T(\tilde{g})-r_{X}^{Z}(g) \in\left(r_{Z}^{X_{n}}\right)^{-1}\left(\frac{1}{2} U_{n}\right)
$$

Because $X_{n} \cap \psi^{m}\left(X_{n}\right)=X_{n} \cap Y=\emptyset$ it follows from the gluing property of a sheaf that there is $f_{n} \in \mathscr{F}\left(X_{n} \cup Y\right)$ with

$$
r_{X_{n} \cup Y}^{X_{n}}\left(f_{n}\right)=r_{X}^{X_{n}}(f) \text { and } r_{X_{n} \cup Y}^{Y}\left(f_{n}\right)=\tilde{g}
$$

By hypothesis b3) there is $h \in \mathscr{F}(X)$ such that

$$
r_{X}^{X_{n} \cup Y}(h)-f_{n} \in\left(r_{X_{n} \cup Y}^{X_{n}}\right)^{-1}\left(\frac{1}{2} U_{n}\right) \cap\left(r_{Z}^{X_{n}} \circ T \circ r_{X_{n} \cup Y}^{Y}\right)^{-1}\left(\frac{1}{2} U_{n}\right)
$$

Thus

$$
\frac{1}{2} U_{n} \ni r_{X_{n} \cup Y}^{X_{n}}\left(r_{X}^{X_{n} \cup Y}(h)-f_{n}\right)=r_{X}^{X_{n}}(h)-r_{X_{n} \cup Y}^{X_{n}}\left(f_{n}\right)=r_{X}^{X_{n}}(h)-r_{X}^{X_{n}}(f),
$$

so that

$$
h \in f+\left(r_{X}^{X_{n}}\right)^{-1}\left(\frac{1}{2} U_{n}\right)
$$


We will show that $C_{w, \psi}^{m}(h) \in g+\left(r_{X}^{X_{n}}\right)^{-1}\left(U_{n}\right)$. Indeed, using (51) we have

$$
\begin{aligned}
& \left(r_{X}^{X_{n}} \circ C_{w, \psi}^{m}\right)(h)-r_{X}^{X_{n}}(g)=\left(r_{Z}^{X_{n}} \circ r_{X}^{Z} \circ C_{w, \psi}^{m}\right)(h) \\
& -\left(r_{Z}^{X_{n}} \circ T\right)(\tilde{g})+\left(r_{Z}^{X_{n}} \circ T\right)(\tilde{g})-r_{Z}^{X_{n}} \circ r_{X}^{Z}(g) \\
& =\left(r_{Z}^{X_{n}} \circ r_{X}^{\left(\psi^{m}\right)^{-1}(Y)} \circ C_{w, \psi}^{m}\right)(h) \\
& -\left(r_{Z}^{X_{n}} \circ T \circ r_{X_{n} \cup Y}^{Y}\right)\left(f_{n}\right)+r_{Z}^{X_{n}}\left(T(\tilde{g})-r_{X}^{Z}(g)\right) \\
& =\left(r_{Z}^{X_{n}} \circ C_{w, \psi,\left(\psi^{m-1}\right)^{-1}(Y)} \circ \ldots \circ C_{w, \psi, Y} \circ r_{X_{n} \cup Y}^{Y}\right. \\
& \left.\circ r_{X}^{X_{n} \cup Y}\right)(h)-\left(r_{Z}^{X_{n}} \circ T \circ r_{X_{n} \cup Y}^{Y}\right)\left(f_{n}\right) \\
& +r_{Z}^{X_{n}}\left(T(\tilde{g})-r_{X}^{Z}(g)\right) \\
& =\left(r_{Z}^{X_{n}} \circ T \circ r_{X_{n} \cup Y}^{Y}\right)\left(r_{X}^{X_{n} \cup Y}(h)-f_{n}\right) \\
& +r_{Z}^{X_{n}}\left(T(\tilde{g})-r_{X}^{Z}(g)\right) \\
& \in \frac{1}{2} U_{n}+\frac{1}{2} U_{n} \subseteq U_{n},
\end{aligned}
$$

so that $C_{w, \psi}^{m}(h) \in g+\left(r_{X}^{X_{n}}\right)^{-1}\left(U_{n}\right)$. Thus we have shown

$$
C_{w, \psi}^{m}\left(f+\left(r_{X}^{X_{n}}\right)^{-1}\left(U_{n}\right)\right) \cap\left(g+\left(r_{X}^{X_{n}}\right)^{-1}\left(U_{n}\right)\right) \neq \emptyset .
$$

That condition a) from the previous proposition is in particular satisfied if $C_{w, \psi}$ has dense range is the content of the next one.

Proposition 3.8. Let $\mathscr{F}$ be a sheaf on $\Omega$ satisfying $(\mathscr{F} 1), X \subseteq \Omega$ be open such that $C_{w, \psi}$ acts locally on $\mathscr{F}(X)$. Assume that $C_{w, \psi}$ has dense range. Then, for every open subset $Y$ of $X$ and any $m \in \mathbb{N}_{0}$

$$
r_{X}^{\left(\psi^{m}\right)^{-1}(Y)}(\mathscr{F}(X)) \subseteq \overline{\left(C_{w, \psi,\left(\psi^{m-1}\right)^{-1}(Y)} \circ \ldots \circ C_{w, \psi, Y}\right)(\mathscr{F}(Y))},
$$

where the closure is taken in $\mathscr{F}\left(\left(\psi^{m}\right)^{-1}(Y)\right)$.

Proof. Fix an open subset $Y$ of $X$ and $m \in \mathbb{N}$. From the hypothesis on the range of $C_{w, \psi}$, the continuity of the restriction map $r_{X}^{\left(\psi^{m}\right)^{-1}(Y)}$, and the commutativity relation (5) it follows

$$
\begin{aligned}
& r_{X}^{\left(\psi^{m}\right)^{-1}(Y)}(\mathscr{F}(X))=r_{X}^{\left(\psi^{m}\right)^{-1}(Y)}\left(\overline{C_{w, \psi}^{m}(\mathscr{F}(X)}\right) \subseteq \overline{r_{X}^{\left(\psi^{m}\right)^{-1}(Y)}\left(C_{w, \psi}^{m}(\mathscr{F}(X))\right)}
\end{aligned}
$$

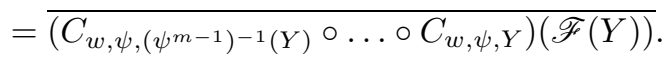

We now come to an almost characterization of weak mixing for weighted composition operators acting locally on $\mathscr{F}(X)$, where $\mathscr{F}$ is a sheaf of functions defined by local properties which satisfies $(\mathscr{F} 1)-(\mathscr{F} 3)$.

Theorem 3.9. Let $\mathscr{F}$ be a sheaf on $\Omega$ satisfying $(\mathscr{F} 1)-(\mathscr{F} 3)$, let $X \subseteq \Omega$ be open, and assume that the weighted composition operator $C_{w, \psi}$ acts locally on $\mathscr{F}(X)$. Then, among the following, $i) \Rightarrow i i) \Rightarrow i v)$. Additionally, if $\mathscr{F}(\Omega)$ is dense in $C(\Omega)$ with respect to the compact-open topology or if $|w(x)| \leq 1$ for all $x \in X$, it holds $i) \Rightarrow i i) \Rightarrow i i i) \Rightarrow i v)$.

i) a) For any $m \in \mathbb{N}_{0}$ and every open, relatively compact $Y \subseteq \psi^{m}(X)$

$$
r_{X}^{\left(\psi^{m}\right)^{-1}(Y)}(\mathscr{F}(X)) \subseteq \overline{\left(C_{w, \psi,\left(\psi^{m-1}\right)^{-1}(Y)} \circ \ldots \circ C_{w, \psi, Y}\right)(\mathscr{F}(Y))},
$$

where the closure is taken in $\mathscr{F}\left(\left(\psi^{m}\right)^{-1}(Y)\right)$.

b) There is an open, relatively compact exhaustion $\left(X_{n}\right)_{n \in \mathbb{N}_{0}}$ of $X$ such that for every $n \in \mathbb{N}_{0}$ there is $m \in \mathbb{N}$ with 
b1) $\psi^{m}\left(X_{n}\right)$ is open.

b2) $X_{n} \cap \psi^{m}\left(X_{n}\right)=\emptyset$.

b3) The restriction map $r_{X}^{X_{n} \cup \psi^{m}\left(X_{n}\right)}$ has dense range.

ii) $C_{w, \psi}$ is weakly mixing on $\mathscr{F}(X)$.

iii) $C_{w, \psi}$ is transitive on $\mathscr{F}(X)$.

iv) a) from i) holds, $w$ has no zeros, $\psi$ is injective and run-away, and in case of $(\mathscr{F} 4)$ with continuously differentiable $w$ and $\psi$, additionally detJ $\psi(x) \neq 0$ for every $x \in X$.

\section{Remark 3.10.}

i) If iv) of the above theorem is valid, $\psi$ is in particular run-away so that b2) is satisfied for any open, relatively compact exhaustion $\left(X_{n}\right)_{n \in \mathbb{N}_{0}}$ of $X$ for suitable $m$.

ii) In case of $\Omega=\mathbb{R}^{d}$, if $\psi$ is injective it follows from Brouwer's Invariance of Domain Theorem (see $[\mathrm{Br}$, Corollary 19.9] for an even stronger result) that for any open subset $Y$ of $X$ and each $m \in \mathbb{N}_{0}$ the set $\psi^{m}(Y)$ is open. Thus, in case of $\Omega=\mathbb{R}^{d}$, the only obstruction against the equivalence of $\mathrm{i}$ ), ii), and iv) in Theorem 3.9 is the existence of a particular open, relatively compact exhaustion $\left(X_{n}\right)_{n \in \mathbb{N}}$ which satisfies b3). In concrete situations, this obstruction is overcome by a suitable approximation result which depending on the concrete sheaf of functions under consideration - can be trivial (e.g. in the case of continuous functions) or highly sophisticated (as in the case of holomorphic functions in several variables [Za]).

iii) Although looking possibly rather deterrering condition a) from item i) in Theorem 3.9 is in most concrete situations fulfilled for zero-free $w$ and injective as well as open (the latter is redundant for $\Omega=\mathbb{R}^{d}$ by Brouwer's Invariance of Domain Theorem) $\psi$ because of the following. Under the stated hypothesis on $w$ and $\psi$ for any open subset $Y$ of $\psi^{m}(X), m \in \mathbb{N}_{0}$, and each $f \in \mathscr{F}\left(\left(\psi^{m}\right)^{-1}(Y)\right)$ the function

$$
\tilde{f}: Y \mapsto \mathbb{K}, y \mapsto\left(\frac{f}{\prod_{j=0}^{m-1} w\left(\psi^{j}(\cdot)\right)}\right) \circ\left(\psi^{m}\right)^{-1}(y)
$$

is a well-defined, continuous function. In case of $\tilde{f} \in \mathscr{F}(Y)$ a straightforward calculation gives

$$
\left(C_{w, \psi,\left(\psi^{m-1}\right)^{-1}(Y)^{\circ}} \ldots \circ C_{w, \psi, Y}\right)(\tilde{f})=f
$$

in $\left(\psi^{m}\right)^{-1}(Y)$. But in many concrete examples, $\tilde{f} \in \mathscr{F}(Y)$ indeed holds - if also $\operatorname{det} J \psi(x) \neq 0$ for all $x \in X$. In case of $\mathscr{F}=C^{r}$ it follows from the fact that if $C_{w, \psi}$ is well-defined then $w \in C^{r}(X)$ and therefore $\frac{1}{w} \in C^{r}(X), \psi$ is $C^{r}$, too, and by the Inverse Function Theorem (see e.g. [Na, Theorem 1.3.2, Remark 1.3.11]) the same holds for $\left(\psi^{m}\right)^{-1}$ so that $\tilde{f} \in C^{r}(Y)$. The same arguments also hold for real analytic or holomorphic functions in several variables (see e.g. again [Na], resp. [FrGr, Theorem 7.5]). Thus, in many concrete examples, condition a) from i) of Theorem 3.9 is redundant in part iv).

Proof of Theorem 3.9. Assume that i) holds and let $V_{j}, W_{j} \subseteq \mathscr{F}(X)$ be non-empty and open, $j=1,2$. We fix $f_{j} \in V_{j}, g_{j} \in W_{j}, j=1,2$. Since by $(\mathscr{F} 1)$ we have $\mathscr{F}(X)=\operatorname{proj}_{\leftarrow n}\left(\mathscr{F}\left(X_{n}\right), r_{X_{n+1}}^{X_{n}}\right)$ topologically, there is $n \in \mathbb{N}$ and an absolute convex zero neighborhood $U_{n}$ in $\mathscr{F}\left(X_{n}\right)$ such that for $j=1,2$ we have

$$
f_{j}+\left(r_{X}^{X_{n}}\right)^{-1}\left(U_{n}\right) \subseteq V_{j} \text { and } g_{j}+\left(r_{X}^{X_{n}}\right)^{-1}\left(U_{n}\right) \subseteq W_{j}
$$


(see e.g. [We, Chapter 3.3]). From hypotheses a) and b) it follows with the aid of Proposition 3.7 that there is $m \in \mathbb{N}$ such that for $j=1,2$ we have

$$
\emptyset \neq C_{w, \psi}^{m}\left(f_{j}+\left(r_{X}^{X_{n}}\right)^{-1}\left(U_{n}\right)\right) \cap\left(g_{j}+\left(r_{X}^{X_{n}}\right)^{-1}\left(U_{n}\right)\right) \subseteq C_{w, \psi}^{m}\left(V_{j}\right) \cap W_{j},
$$

so that ii) holds.

ii) obviously implies iii).

If ii) is satisfied (respectively if iii) is satisfied and $\mathscr{F}(\Omega)$ is dense in $C(\Omega)$ or $|w| \leq 1) C_{w, \psi}$ has in particular dense range so that condition a) in i) follows from Proposition 3.8 while the rest of the properties listed in iv) follow from Proposition 3.4 .

Concrete applications of Theorem [3.9 will be postponed to sections [4, 5] and 6. We next come to an almost characterization of mixing of weighted composition operators on local function spaces.

Theorem 3.11. Let $\mathscr{F}$ be a sheaf on $\Omega$ satisfying $(\mathscr{F} 1)-(\mathscr{F} 3)$, let $X \subseteq \Omega$ be open, and assume that the weighted composition operator $C_{w, \psi}$ acts locally on $\mathscr{F}(X)$. Then, among the following, $i) \Rightarrow i i) \Rightarrow i i i)$ :

i) a) For any $m \in \mathbb{N}_{0}$ and every open, relatively compact $Y \subseteq \psi^{m}(X)$

$$
r_{X}^{\left(\psi^{m}\right)^{-1}(Y)}(\mathscr{F}(X)) \subseteq \overline{\left(C_{w, \psi,\left(\psi^{m-1}\right)^{-1}(Y)} \circ \ldots \circ C_{w, \psi, Y}\right)(\mathscr{F}(Y))},
$$

where the closure is taken in $\mathscr{F}\left(\left(\psi^{m}\right)^{-1}(Y)\right)$.

b) There is an open, relatively compact exhaustion $\left(X_{n}\right)_{n \in \mathbb{N}_{0}}$ of $X$ such that for every $n \in \mathbb{N}_{0}$ there is $M \in \mathbb{N}$ with

b1) $\psi^{m}\left(X_{n}\right)$ is open for all $m \geq M$.

b2) $X_{n} \cap \psi^{m}\left(X_{n}\right)=\emptyset$ for all $m \geq M$.

b3) The restriction map $r_{X}^{X_{n} \cup \psi^{m}\left(X_{n}\right)}$ has dense range for all $m \geq M$.

ii) $C_{w, \psi}$ is mixing on $\mathscr{F}(X)$.

iii) a) from i) holds, $w$ has no zeros, $\psi$ is injective and strong run-away, and in case of $(\mathscr{F} 4)$ with continuously differentiable $w$ and $\psi$, additionally $\operatorname{det} J \psi(x) \neq 0$ for every $x \in X$.

Proof. In order to show that i) implies ii) let $V, W \subseteq \mathscr{F}(X)$ be open and non-empty. As in the proof of the implication "i) $\Rightarrow$ ii)" of Theorem [3.9. let $f \in V, g \in W$, and choose $n \in \mathbb{N}$ and an absolutely convex zero neighborhood $U_{n}$ in $\mathscr{F}\left(X_{n}\right)$ such that

$$
f+\left(r_{X}^{X_{n}}\right)^{-1}\left(U_{n}\right) \subseteq V \text { and } g+\left(r_{X}^{X_{n}}\right)^{-1}\left(U_{n}\right) \subseteq W .
$$

From the hypotheses a), b1)-b3) it follows together with Proposition 3.7 that there is $M \in \mathbb{N}$ such that for any $m \geq M$

$$
\emptyset \neq C_{w, \psi}^{m}\left(f+\left(r_{X}^{X_{n}}\right)^{-1}\left(U_{n}\right)\right) \cap\left(g+\left(r_{X}^{X_{n}}\right)^{-1}\left(U_{n}\right)\right) \subseteq C_{w, \psi}^{m}(V) \cap W,
$$

so that $C_{w, \psi}$ is mixing.

If on the other hand $C_{w, \psi}$ is mixing, $C_{w, \psi}$ is in particular weakly mixing so that by Theorem 3.9 we only have to show that $\psi$ is strong run away. Assume that this is not the case, i.e. that there is a compact subset $K$ of $X$ and a strictly increasing sequence of natural numbers $\left(m_{l}\right)_{l \in \mathbb{N}}$ such that

$$
\forall l \in \mathbb{N} \exists x_{l} \in K: \psi^{m_{l}}\left(x_{l}\right) \in K .
$$

By $(\mathscr{F} 2)$ there is $f_{K} \in \mathscr{F}(X)$ such that $f_{K}(x) \neq 0$ for all $x$ in $K$. Then

$$
\alpha:=\inf _{x \in K}\left|f_{K}(x)\right|>0 \text { and } \beta:=\sup _{x \in K}\left|f_{K}(x)\right|<\infty
$$

and the set

$$
U:=\left\{g \in \mathscr{F}(X) ; \forall x \in K: \frac{\alpha}{2}<|g(x)|<2 \beta\right\}
$$


contains $f_{K}$ and is open with respect to the compact-open topology and is therefore an open neighborhood of $f_{K}$ in $\mathscr{F}(X)$. The set

$$
V:=\left\{g \in \mathscr{F}(X) ; \sup _{x \in K}|g(x)|<\frac{\alpha^{2}}{8 \beta}\right\}
$$

is open in $\mathscr{F}(X)$, too, and contains the zero function. Since $C_{w, \psi}$ is mixing, there is $M \in \mathbb{N}$ such that

$$
\forall m \geq M: C_{w, \psi}^{m}(U) \cap U \neq \emptyset \text { and } C_{w, \psi}^{m}(U) \cap V \neq \emptyset .
$$

Now we fix $l \in \mathbb{N}$ with $m_{l}>M$ and pick $f \in U$ with $C_{w, \psi}^{m_{l}}(f) \in U$ as well as $g \in U$ with $C_{w, \psi}^{m_{l}}(g) \in V$. As in the proof of Proposition $3.4 \mathrm{v}$ ) under the additional assumption $\mathrm{v}-2$ ) one deduces the contradiction

$$
\frac{\alpha}{4 \beta}<\left|\prod_{j=0}^{m_{l}-1} w\left(\psi^{j}\left(x_{l}\right)\right)\right|<\frac{\alpha}{4 \beta} .
$$

Thus, $\psi$ is strong run-away.

\section{DyNAMiCS OF WEIGHTED COMPOSITION OPERATORS ON CONCRETE LOCAL FUNCTION SPACES}

As a first application of the results from the previous section we next show how to use them to recover characterizations of transitivity/hypercyclicity and mixing of weighted composition operators on various concrete functions spaces obtained by several authors or which we assume to be well-known (and add a slight generalization here and there).

4.1. Continuous functions. For an arbitrary locally compact, $\sigma$-compact, noncompact Hausdorff space $\Omega$ the sheaf of continuous functions $C$ satisfies properties $(\mathscr{F} 1)-(\mathscr{F} 3)$ as explained in example 2.3 i). Clearly, for an arbitrary open subset $X \subseteq \Omega, w \in C(X)$, and continuous $\psi: X \rightarrow X$ the weighted composition operator $C_{w, \psi}$ is well-defined on $C(X)$ and acts locally on $C(X)$.

Recall that locally compact spaces are completely regular and that by Wa, Theorem 5] for a completely regular topological space $Z$ the space $C(Z)$ equipped with the compact-open topology is separable, if and only if $Z$ has a separable metrizable compression, i.e. if and only if $Z$ has a weaker separable metrizable topology. Thus, in case the open subset $X$ of $\Omega$ below has a weaker separable metrizable topology, part a) of the next result characterizes hypercyclicity of $C_{w, \psi}$ on $C(X)$.

Corollary 4.1. Let $\Omega$ be a locally compact, $\sigma$-compact, non-compact Hausdorff space, $X \subseteq \Omega$ be open, $w \in C(X)$ and $\psi: X \rightarrow X$ be continuous. If $\Omega \neq \mathbb{R}^{d}$ we assume additionally that $\psi$ is open.

a) The following are equivalent.

i) $C_{w, \psi}$ is weakly mixing on $C(X)$.

ii) $C_{w, \psi}$ is transitive on $C(X)$.

iii) $w$ has no zeros, $\psi$ is injective and run-away.

b) The following are equivalent.

i) $C_{w, \psi}$ is mixing on $C(X)$.

ii) $w$ has no zeros, $\psi$ is injective and strong run-away.

Proof. We first prove a). Clearly, i) implies ii) and by Theorem 3.9, ii) implies iii). Now, if iii) is satisfied it follows from Remark 3.10 that condition i) a) from Theorem 3.9 is fulfilled. Let $\left(X_{n}\right)_{n \in \mathbb{N}_{0}}$ be an arbitrary open, relatively compact exhaustion of $X$. If $\Omega=\mathbb{R}^{d}$ it follows from Brouwer's Invariance of Domain Theorem, $\psi^{m}$ is 
an open mapping on $X$ for any $m$, in particular, for every $n \in \mathbb{N}_{0}$ and any $m \in \mathbb{N}$, $\psi^{m}\left(X_{n}\right)$ is an open subset of $X$. In case of $\Omega \neq \mathbb{R}^{d}$ the same follows from the hypotheses on $\psi$. For fixed $n \in \mathbb{N}_{0}$, since $\psi$ is run-away, there is $m \in \mathbb{N}$ such that

$$
X_{n} \cap \psi^{m}\left(X_{n}\right)=\emptyset \text {. }
$$

Let $K$ be a compact subset of $X_{n} \cup \psi^{m}\left(X_{n}\right)$. Since $X$ is a locally compact Hausdorff space, $X$ is normal and therefore in particular $X$ is regular. Thus, for every $x \in K$ there is an open neighborhood $V_{x}$ of $x$ such that $\overline{V_{x}} \subseteq X_{n} \cup \psi^{m}\left(X_{n}\right)$. Using the compactness of $K$ we deduce from this that there is an open neighborhood $V$ of $K$ such that $\bar{V} \subseteq X_{n} \cup \psi^{m}\left(X_{n}\right)$. By Urysohn's Lemma [En, Theorem 1.5.11] there is $h \in C(X)$ such that $h=1$ on $K$ and $h=0$ on $X \backslash V$, thus $\operatorname{supp} h \subseteq \bar{V}$. Now if $g \in C\left(X_{n} \cup \psi^{m}\left(X_{n}\right)\right)$ we obtain a continuous function $f$ on $X$ with $f_{\mid K}=g$ by extending $h g$ by zero outside of $X_{n} \cup \psi^{m}\left(X_{n}\right)$. Since $K \subset X_{n} \cup \psi^{m}\left(X_{n}\right)$ was an arbitary compact set it follows that $r_{X}^{X_{n} \cup \psi^{m}\left(X_{n}\right)}$ has dense range. Hence, condition i) b) from Theorem 3.9 is also satisfied, so that iii) implies i).

Referring to Theorem 3.11 instead of Theorem 3.9 the proof of $b$ ) is mutatis mutandis the same as the proof of part a).

4.2. $C^{r}$-functions on open subsets of $\mathbb{R}^{d}$. Let $\mathscr{F}$ be the sheaf $C^{r}$ of $r$ times continuously differentiable functions on $\mathbb{R}^{d}$ (equipped with the topology of local uniform convergence of partial derivatives of order less than $r+1)$. Then $C^{r}$ satisfies properties $(\mathscr{F} 1)-(\mathscr{F} 4)$ as explained in example 2.3 ii) and $C^{r}(X)$ is a separable Fréchet space for every open subset $X \subseteq \mathbb{R}^{d}$. Clearly, for an arbitrary open subset $X \subseteq \mathbb{R}^{d}, w \in C^{r}(X)$, and a $C^{r}$-function $\psi: X \rightarrow X$ the weighted composition operator $C_{w, \psi}$ is well-defined on $C^{r}(X)$ and acts locally on $C^{r}(X)$. The next application of the results from the previous section gives the results obtained by Przestacki in $[\mathrm{Pr}]$ in case of $r=\infty$.

Corollary 4.2. Let $X \subseteq \mathbb{R}^{d}$ be open, $r \in \mathbb{N} \cup\{\infty\}, w \in C^{r}(X)$ and $\psi: X \rightarrow X$ be a $C^{r}$-function.

a) The following are equivalent.

i) $C_{w, \psi}$ is weakly mixing on $C^{r}(X)$.

ii) $C_{w, \psi}$ is hypercyclic on $C^{r}(X)$.

iii) $w$ has no zeros, $\psi$ is injective, run-away, and detJ $\psi(x) \neq 0$ for all $x \in X$.

b) The following are equivalent.

i) $C_{w, \psi}$ is mixing on $C^{r}(X)$.

ii) $w$ has no zeros, $\psi$ is injective, strong run-away, and det $\psi(x) \neq 0$ for all $x \in X$.

Proof. We first prove a). Since $C^{r}(X)$ is a separable Fréchet space, by Birkhoff's transitivity criterion, hypercyclicity of $C_{w, \psi}$ is equivalent to transitivity. Thus, i) implies ii) and since polynomials, a fortiori $C^{r}\left(\mathbb{R}^{d}\right)$, are dense in $C\left(\mathbb{R}^{d}\right)$ (see e.g. $\mathrm{Tr}$, Chapter 15, Corollary 4]) by Theorem [3.9, ii) implies iii). Now, if iii) is satisfied it follows from Remark [3.10] that condition i) a) from Theorem [3.9] is fulfilled. Let $\left(X_{n}\right)_{n \in \mathbb{N}_{0}}$ be an arbitrary open, relatively compact exhaustion of $X$. Because $\operatorname{det} J(x) \neq 0$ for all $x \in X$ it follows together with the injectivity of $\psi$ that $\psi^{m}$ is an open mapping (see e.g. $\left[\mathrm{Na}\right.$, Theorem 1.3.2]). In particular, for every $n \in \mathbb{N}_{0}$ and any $m \in \mathbb{N}, \psi^{m}\left(X_{n}\right)$ is an open subset of $X$. For fixed $n \in \mathbb{N}_{0}$, since $\psi$ is run-away, there is $m \in \mathbb{N}$ such that

$$
X_{n} \cap \psi^{m}\left(X_{n}\right)=\emptyset .
$$

Let $K$ be a compact subset of $X_{n} \cup \psi^{m}\left(X_{n}\right)$ and $V$ an open $K$ such that $\bar{V} \subseteq$ $X_{n} \cup \psi^{m}\left(X_{n}\right)$. Then there is $h \in C^{\infty}(X)$ such that $h=1$ on $K$ and $\operatorname{supp} h \subseteq \bar{V}$. Now if $g \in C^{\infty}\left(X_{n} \cup \psi^{m}\left(X_{n}\right)\right)$ we obtain a smooth function $f$ on $X$ with $f_{\mid K}=g$ 
by extending $h g$ by zero outside of $X_{n} \cup \psi^{m}\left(X_{n}\right)$. Since $K \subseteq X_{n} \cup \psi^{m}\left(X_{n}\right)$ was an arbitary compact set we conclude that $r_{X}^{X_{n} \cup \psi^{m}\left(X_{n}\right)}$ has dense range so that i) b) from Theorem 3.9 is fulfilled. Thus, iii) implies i).

Referring again to Theorem 3.11 instead of Theorem 3.9 the proof of b) is mutatis mutandis the same as the proof of part a).

4.3. Real analytic functions on open subsets of $\mathbb{R}^{d}$. Let $\mathscr{F}$ be the sheaf $\mathscr{A}$ of real analytic functions on $\mathbb{R}^{d}$ (equipped with its natural topology, see Example $2.3 \mathrm{v}))$. Then $\mathscr{A}$ satisfies properties $(\mathscr{F} 1)-(\mathscr{F} 4)$ as explained in Examples $2.3 \mathrm{v})$ and clearly, for an arbitrary open subset $X \subseteq \mathbb{R}^{d}, w \in \mathscr{A}(X)$, and real analytic $\psi: X \rightarrow X$ the weighted composition operator $C_{w, \psi}$ is well-defined on $\mathscr{A}(X)$ and acts locally on $\mathscr{A}(X)$. For the special case of $w=1$, the equivalence of ii) and iii) in part a) of our next application of the results from the previous section was obtained by Bonet and Domański in BoDo, Theorem 2.3].

Corollary 4.3. Let $X \subseteq \mathbb{R}^{d}$ be open, $w \in \mathscr{A}(X)$ and $\psi: X \rightarrow X$ be real analytic.

a) The following are equivalent.

i) $C_{w, \psi}$ is weakly mixing on $\mathscr{A}(X)$.

ii) $C_{w, \psi}$ is transitive on $\mathscr{A}(X)$.

iii) $w$ has no zeros, $\psi$ is injective, run-away, and detJ $\psi(x) \neq 0$ for all $x \in X$.

b) The following are equivalent.

i) $C_{w, \psi}$ is mixing on $\mathscr{A}(X)$.

ii) $w$ has no zeros, $\psi$ is injective, strong run-away, and $\operatorname{det} J \psi(x) \neq 0$ for all $x \in X$.

Proof. As before, we first prove a). Obviously, i) implies ii) and because polynomials are dense in $C\left(\mathbb{R}^{d}\right)$ (see e.g. $\operatorname{Tr}$, Chapter 15, Corollary 4]) by Theorem [3.9, ii) implies iii). Now, if iii) is satisfied it follows from Remark 3.10 that condition i) a) from Theorem 3.9 is fulfilled. Let $\left(X_{n}\right)_{n \in \mathbb{N}_{0}}$ be an arbitrary open, relatively compact exhaustion of $X$. As in the proof of Corollary 4.2 it follows that for every $n \in \mathbb{N}_{0}$ and any $m \in \mathbb{N}, \psi^{m}\left(X_{n}\right)$ is an open subset of $X$. For fixed $n \in \mathbb{N}_{0}$, since $\psi$ is run-away, there is $m \in \mathbb{N}$ such that

$$
X_{n} \cap \psi^{m}\left(X_{n}\right)=\emptyset,
$$

so that b1) and b2) from i) in Theorem 3.9 are fulfilled.

In order to show that b3) is fulfilled, too, let $f \in \mathscr{A}\left(X_{n} \cup \psi^{m}\left(X_{n}\right)\right)$ be arbitrary and let $V$ be any neighborhood of $f$ in $\mathscr{A}\left(X_{n} \cup \psi^{m}\left(X_{n}\right)\right)$. By the definition of the topology on $\mathscr{A}\left(X_{n} \cup \psi^{m}\left(X_{n}\right)\right)$ there is a compact subset $K$ of $X_{n} \cup \psi^{m}\left(X_{n}\right)$ and a complex neighborhood $W_{0} \subseteq \mathbb{C}^{d}$ of $K$ such that $f$ extends to a holomorphic function on $W_{0}$ and such that for every complex neighborhood $W$ of $K$ with $\bar{W} \subseteq W_{0}$ there is $\delta>0$ with

$$
\left\{g \in \mathscr{H}^{\infty}(W) ;\|f-g\|_{\infty, W}<\delta\right\} \subseteq V,
$$

where $\|\cdot\|_{\infty, W}$ denotes the supremum norm over $W$. Because compact subsets of $\mathbb{R}^{d}$ are polynomially convex in $\mathbb{C}^{d}$ it follows from $[$ Hö2, Theorem 2.7.7] that for any relatively compact, complex neighborhood $W$ of $K$ with $\bar{W} \subseteq W_{0}$ there is a (holomorphic) polynomial $p$ such that $\|f-p\|_{\infty, W}<\delta$. In particular, $p_{\mid X} \in$ $V \cap \mathscr{A}(X)$. By the arbitrariness of $V$ and $f$ it follows that the restriction map $r_{X}^{X_{n} \cup \psi^{m}\left(X_{n}\right)}$ has dense range so that b3) of part i) in Theorem 3.9 is indeed fulfilled. Now, by the same theorem, iii) implies i) so that a) is proved.

The proof of part b) is again done as the proof of a) by referring to Theorem 3.11 instead of Theorem 3.9 . 
A generalization of the setting of real analytic functions is given in the next section.

\section{Spaces of ultradifferentiable functions}

In this section we consider as our function spaces defined by local properties spaces of ultradifferentiable functions on open subsets of $\mathbb{R}^{d}$, both of Roumieu type and Beurling type, and both quasianalytic classes as well as non-quasianalytic classes.

There are at least two ways to define spaces of ultradifferentiable functions. Classical Denjoy-Carleman classes are defined as smooth functions satisfying certain growth conditions on their Taylor coefficients while it was observed by Beurling Beu (see also Björck $[\mathrm{Bj}]$ ) that one can also use decay properties with respect to a weight function of the Fourier transform of compactly supported smooth functions for this purpose as well. The latter approach was vastly generalized by Braun, Meise, and Taylor in BrMeTa. It is their approach to ultradifferentiable functions which we will follow here. For a comparison of these two approaches, see [BoMeMe]; see also the article RaSc1 by Rainer and Schindl.

Recall that a continuous increasing function $\omega:[0, \infty) \rightarrow[0, \infty)$ satisfying $\omega_{\mid[0,1]}=0$ is called a weight function if the following properties hold:

$(\alpha)$ There is $K \geq 1$ such that $\omega(2 t) \leq K(1+\omega(t))$ for all $t \geq 0$.

( $\beta) \omega(t)=O(t)$ as $t$ tends to infinity.

$(\gamma) \log (1+t)=o(\omega(t))$ as $t$ tends to infinity.

$(\delta) \varphi:[0, \infty) \rightarrow[0, \infty), \varphi(x):=\omega\left(e^{x}\right)$ is convex.

Recall that a weight function $\omega$ is called quasianalytic if it satisfies the property

(q) $\int_{1}^{\infty} \frac{\omega(t)}{t^{2}} d t=\infty$

A weight function which does not satisfy $(q)$ is called non-quasianalytic. Because of $(\gamma)$ and $(\delta)$, for a weight function $\omega$ and $\varphi$ as in $(\delta)$, the Young conjugate $\varphi^{*}$ of $\varphi$

$$
\varphi^{*}:[0, \infty) \rightarrow[0, \infty), \varphi^{*}(y):=\sup _{x \geq 0}(y x-\varphi(x))
$$

is well-defined, convex, increasing, and satisfies $\varphi^{*}(0)=0, \lim _{y \rightarrow \infty} \frac{y}{\varphi^{*}(y)}=0$, and $\left(\varphi^{*}\right)^{*}=\varphi$. For a weight function $\omega$ and an open $X \subseteq \mathbb{R}^{d}$ we define

$$
\begin{aligned}
& \mathscr{E}_{(\omega)}(X):=\left\{f \in C^{\infty}(X): \forall K \subseteq X \text { compact } \forall m \in \mathbb{N}:\right. \\
&\left.\|f\|_{(\omega), K, m}:=\sup _{x \in K} \sup _{\alpha \in \mathbb{N}_{0}^{d}}\left|\partial^{\alpha} f(x)\right| \exp \left(-m \varphi^{*}\left(\frac{|\alpha|}{m}\right)\right)<\infty\right\}
\end{aligned}
$$

and

$$
\begin{aligned}
& \mathscr{E}_{\{\omega\}}(X):=\left\{f \in C^{\infty}(X): \forall K \subseteq X \text { compact } \exists m \in \mathbb{N}:\right. \\
&\left.\|f\|_{\{\omega\}, K, m}:=\sup _{x \in K} \sup _{\alpha \in \mathbb{N}_{0}^{d}}\left|\partial^{\alpha} f(x)\right| \exp \left(-\frac{1}{m} \varphi^{*}(m|\alpha|)\right)<\infty\right\} .
\end{aligned}
$$

The elements of $\mathscr{E}_{(\omega)}(X)$, resp. $\mathscr{E}_{\{\omega\}}(X)$, are called $\omega$-ultradifferentiable functions of Beurling type on $X$, resp. $\omega$-ultradifferentiable functions of Roumieu type on $X$. Obviously, $\mathscr{E}_{(\omega)}(X) \subseteq \mathscr{E}_{\{\omega\}}(X)$ and clearly, $\mathscr{E}_{(\omega)}$ and $\mathscr{E}_{\{\omega\}}$ are sheaves on $\mathbb{R}^{d}$. $\mathscr{E}_{(\omega)}(X)$ contains non-trivial functions with compact support for some non-empty open $X$ if and only if $\omega$ is non-quasianalytic.

Prominent examples of weights are $\omega_{\beta}(t)=t^{\beta}$ with $0<\beta<1$ for which $\varphi^{*}(y)=y / \beta \log (y / e \beta)$ so that $\exp \left(-\varphi^{*}(|\alpha| \lambda) / \lambda\right)=(\lambda / \beta)^{-|\alpha| / \beta}(|\alpha| / e)^{-|\alpha| / \beta}$. By Stirling's formula $\mathscr{E}_{\left\{\omega_{\beta}\right\}}(X)$ is the classical Gevrey class of exponent $1 / \beta, \Gamma^{1 / \beta}(X)$, and $\mathscr{E}_{\left(\omega_{\beta}\right)}(X)$ is the so-called small Gevrey class of exponent $1 / \beta, \gamma^{1 / \beta}(X)$. The 
spaces $\Gamma^{1 / \beta}(X)$ play an important role in the regularity theory of solutions of hypoelliptic partial differential equations, see [Hö1, Section 11.4].

Moreover, for the weight function $\omega(t)=t$ the corresponding Roumieu space $\mathscr{E}_{\{\omega\}}(X)$ coincides with $\mathscr{A}(X)$ while the corresponding Beurling space $\mathscr{E}_{(\omega)}(X)$ consist of the restrictions to $X$ of functions from $\mathscr{H}\left(\mathbb{C}^{d}\right)$.

As usual, $\mathscr{E}_{(\omega)}(X)$ will be equipped with the locally convex topology induced by the family $\left\{\|\cdot\|_{(\omega), K, m} ; K \subseteq X\right.$ compact, $\left.m \in \mathbb{N}\right\}$ of seminorms, and $\mathscr{E}_{\{\omega\}}(X)$ will be topologized as $\operatorname{proj}_{\leftarrow Y} \operatorname{ind}_{m \rightarrow \infty} \mathscr{E}_{\{\omega\}}(Y, m)$, where for each open, relatively compact subset $Y$ of $X$

$$
\begin{aligned}
\mathscr{E}_{\{\omega\}}(Y, m):= & \left\{f \in C^{\infty}(Y) ;\right. \\
& \left.\|f\|_{\{\omega\}, Y, m}:=\sup _{x \in Y} \sup _{\alpha \in \mathbb{N}_{0}^{d}}\left|\partial^{\alpha} f(x)\right| \exp \left(-\frac{1}{m} \varphi^{*}(m|\alpha|)\right)<\infty\right\}
\end{aligned}
$$

endowed with the norm $\|\cdot\|_{\{\omega\}, Y, m}$ is a Banach space.

Proposition 5.1. Let $\omega$ be a weight function. Equipped with their usual locally convex topologies the sheaves $\mathscr{E}_{(\omega)}$ and $\mathscr{E}\{\omega\}$ on $\mathbb{R}^{d}$ both satisfy properties $(\mathscr{F} 1)-$ $(\mathscr{F} 4)$.

Proof. It is well-known that for open $X \subseteq \mathbb{R}^{d}$ the space $\mathscr{E}_{(\omega)}(X)$ is a (nuclear) Fréchet space, BrMeTa, Proposition 4.9]. Obviously, point evaluations $\delta_{x}$ are continuous linear functionals on $\mathscr{E}_{(\omega)}(X)$ for any $x \in X$. Therefore, as observed in Remark 2.2 i) the sheaf $\mathscr{E}_{(\omega)}$ on $\mathbb{R}^{d}$ satisfies $(\mathscr{F} 1)$. Moreover, since $\mathscr{E}_{(\omega)}(X)$ is closed under differentiation and since polynomials obviously belong to $\mathscr{E}_{(\omega)}(X)$, properties $(\mathscr{F} 2)-(\mathscr{F} 4)$ are fulfilled, too.

Clearly, in the definition of the topology of $\mathscr{E}_{\{\omega\}}(X)$ it is enough to take the projective limit with respect to an open, relatively compact exhaustion $\left(Y_{n}\right)_{n \in \mathbb{N}}$ of $X$. Therefore, being the projective limit of a sequence of $L B$-spaces it follows that $\mathscr{E}_{\{\omega\}}(X)$ is webbed. It has been shown recently by Debrouwere and Vindas DeVi, Proposition 3.2] that the space of ultradifferentiable functions of Roumieu type is ultrabornological.

Let $\left(X_{n}\right)_{n \in \mathbb{N}_{0}}$ be an open, relatively compact exhaustion of $X$. In order to show that the continuous bijection

$$
i: \mathscr{E}_{\{\omega\}}(X) \rightarrow \operatorname{proj}_{\leftarrow n}\left(\mathscr{E}_{\{\omega\}}\left(X_{n}\right), r_{X_{n+1}}^{X_{n}}\right), f \mapsto\left(r_{X}^{X_{n}}(f)\right)_{n \in \mathbb{N}_{0}}
$$

is open, let $V$ be an arbitrary zero neighborhood in $\mathscr{E}_{\{\omega\}}(X)$. By the definition of the topology on $\mathscr{E}_{\{\omega\}}(X)$ there is $n \in \mathbb{N}$ and zero neighborhood $U_{n}$ in $\operatorname{ind}_{m \rightarrow \infty} \mathscr{E}_{\{\omega\}}\left(X_{n}, m\right)$ such that $\rho_{n}^{-1}\left(U_{n}\right) \subseteq V$ where

$$
\rho_{n}: \mathscr{E}_{\{\omega\}}(X) \rightarrow \operatorname{ind}_{m \rightarrow \infty} \mathscr{E}_{\{\omega\}}\left(X_{n}, m\right), f \mapsto f_{\mid X_{n}} .
$$

With the continuous

$$
\tilde{\rho}_{n+1}: \mathscr{E}_{\{\omega\}}\left(X_{n+1}\right) \rightarrow \operatorname{ind}_{m \rightarrow \infty} \mathscr{E}_{\{\omega\}}\left(X_{n}, m\right), f \mapsto f_{\mid X_{n}}
$$

it follows that $\tilde{\rho}_{n+1}^{-1}\left(U_{n}\right)$ is a zero neighborhood in $\mathscr{E}_{\{\omega\}}\left(X_{n+1}\right)$ for which

$$
\tilde{\rho}_{n+1}^{-1}\left(U_{n}\right) \cap r_{X}^{X_{n+1}}\left(\mathscr{E}_{\{\omega\}}(X)\right)=r_{X}^{X_{n+1}}\left(\rho_{n}^{-1}\left(U_{n}\right)\right) \subseteq r_{X}^{X_{n+1}}(V) .
$$

For $k \in \mathbb{N}_{0}$, let

$$
\pi_{k}: \operatorname{proj}_{\leftarrow n}\left(\mathscr{E}_{\{\omega\}}\left(X_{n}\right), r_{X_{n+1}}^{X_{n}}\right) \rightarrow \mathscr{E}_{\{\omega\}}\left(X_{k}\right),\left(f_{n}\right)_{n \in \mathbb{N}_{0}} \mapsto f_{k}
$$

so that

$$
\begin{aligned}
i(V) & =\pi_{n+1}^{-1}\left(r_{X}^{X_{n+1}}(V)\right) \supseteq \pi_{n+1}^{-1}\left(\tilde{\rho}_{n+1}^{-1}\left(U_{n}\right) \cap r_{X}^{X_{n+1}}\left(\mathscr{E}_{\{\omega\}}(X)\right)\right) \\
& =\pi_{n+1}^{-1}\left(\tilde{\rho}_{n+1}^{-1}\left(U_{n}\right)\right) .
\end{aligned}
$$


Since $\tilde{\rho}_{n+1}^{-1}\left(U_{n}\right)$ is a zero neighborhood in $\mathscr{E}_{\{\omega\}}\left(X_{n+1}\right)$ the above inclusion implies that $i(V)$ is a zero neigborhood in $\operatorname{proj}_{\leftarrow n}\left(\mathscr{E}\{\omega\}\left(X_{n}\right), r_{X_{n+1}}^{X_{n}}\right)$ so that $i$ is open and the sheaf $\mathscr{E}\{\omega\}$ satisfies $(\mathscr{F} 1)$.

Properties $(\mathscr{F} 2)-(\mathscr{F} 4)$ of $\mathscr{E}_{\{\omega\}}$ follow again from the fact that $\mathscr{E}\{\omega\}(X)$ is closed under differentiation and contains all polynomials.

Since for arbitrary weight functions $\omega$ the spaces $\mathscr{E}_{(\omega)}(X)$ and $\mathscr{E}_{\{\omega\}}(X)$ are locally convex algebras (see BrMeTa, Proposition 4.4]) it follows that a weighted composition operator $C_{w, \psi}$ is well-defined on $\mathscr{E}_{(\omega)}(X)$ resp. $\mathscr{E}_{\{\omega\}}(X)$ whenever the weight $w$ belongs to the ultradifferentiable class and additionally, the composition with $\psi$ defines a continuous linear operator on $\mathscr{E}_{(\omega)}(X)$, resp. $\mathscr{E}_{\{\omega\}}(X)$. For non-quasianalytic weight functions $\omega$ this has been characterized by Fernández and Galbis in FeGa] while Rainer and Schindl extended this characterization, among others, to more general weight functions in RaSc1, see also RaSc2. We define for a weight function $\omega$ property

$\left(\alpha_{0}\right) \exists C>0, t_{0}>0 \forall \lambda \geq 1, t \geq t_{0}: \omega(\lambda t) \leq C \lambda \omega(t)$.

Property $\left(\alpha_{0}\right)$ characterizes when composition with a smooth function $\psi: X \rightarrow X$ with components $\psi_{j}, 1 \leq j \leq d$, all belonging to $\mathscr{E}_{\{\omega\}}(X)$ defines a continuous linear operator on $\mathscr{E}_{\{\omega\}}(X)$. If $\omega(t)=o(t)$ as $t$ tends to infinity, $\left(\alpha_{0}\right)$ characterizes when composition with a smooth function $\psi: X \rightarrow X$ with components $\psi_{j}, 1 \leq j \leq d$, all belonging to $\mathscr{E}_{(\omega)}(X)$ is a continuous linear operator on $\mathscr{E}_{(\omega)}(X)$. Thus, under these conditions, $C_{w, \psi}$ is then a well-defined, continuous linear operator which then also acts locally on $\mathscr{E}_{(\omega)}(X)$ resp. $\mathscr{E}_{\{\omega\}}(X)$.

Theorem 5.2. Let $\omega$ be a weight function satisfying $\left(\alpha_{0}\right)$ and such that $\omega(t)=o(t)$ as $t$ tends to infinity. Moreover, let $X \subseteq \mathbb{R}^{d}$ be open, $w \in \mathscr{E}_{(\omega)}(X)$, and $\psi: X \rightarrow X$ be smooth such that $\psi_{j} \in \mathscr{E}_{(\omega)}(X)$ for all $1 \leq j \leq d$.

a) The following are equivalent.

i) $C_{w, \psi}$ is weakly mixing on $\mathscr{E}_{(\omega)}(X)$.

ii) $C_{w, \psi}$ is hypercyclic on $\mathscr{E}_{(\omega)}(X)$.

iii) $w$ has no zeros, $\psi$ is injective, run-away, and detJ $\psi(x) \neq 0$ for all $x \in X$.

b) The following are equivalent.

i) $C_{w, \psi}$ is mixing on $\mathscr{E}_{(\omega)}(X)$.

ii) $w$ has no zeros, $\psi$ is injective, strong run-away, and $\operatorname{det} J \psi(x) \neq 0$ for all $x \in X$.

Proof. By a result due to Heinrich and Meise HeMe, Proposition 3.2] $\mathscr{H}\left(\mathbb{C}^{d}\right)$ is dense in $\mathscr{E}_{(\omega)}(X)$. In particular, (holomorphic) polynomials are dense in $\mathscr{E}_{(\omega)}(X)$ implying that the latter Fréchet space is separable. Because polynomials are contained in $\mathscr{E}_{(\omega)}\left(\mathbb{R}^{d}\right)$ it follows that the latter space is dense in $C\left(\mathbb{R}^{d}\right)$. By Theorem 3.9 it thus follows that i) implies ii) and that ii) implies iii) in part a).

If iii) in a) is satisfied, it follows from the hypotheses on $\omega$ and RaSc2, Theorem 4] that $\frac{1}{w} \in \mathscr{E}_{(\omega)}(X)$ and that for any $m \in \mathbb{N}$ the components of the smooth function $\left(\psi^{m}\right)^{-1}: X \rightarrow X$ belong to $\mathscr{E}_{(\omega)}(X)$. Therefore, applying again RaSc2, Theorem 4] it follows that for every open subset $Y$ of $\psi^{m}(X)$ and any $f \in \mathscr{E}_{(\omega)}\left(\left(\psi^{m}\right)^{-1}(Y)\right)$ the function

$$
\tilde{f}: Y \rightarrow \mathbb{K}, y \mapsto\left(\frac{f}{\prod_{j=0}^{m-1} w\left(\psi^{j}(\cdot)\right)}\right) \circ\left(\psi^{m}\right)^{-1}(y)
$$

belongs to $\mathscr{E}_{(\omega)}(Y)$. As detailed in Remark 3.10 iii) this implies that condition a) of part i) of Theorem 3.9 is satisfied. Moreover, because $\psi$ is run-away and $\operatorname{det} J \psi(x) \neq 0$ for every $x \in X$ it follows that conditions b1) and b2) from part i) of Theorem 3.9 are fulfilled for any open, relatively compact exhaustion $\left(X_{n}\right)_{n \in \mathbb{N}}$ 
of $X$. Finally, applying HeMe, Proposition 3.2] once more it follows in particular that condition b3) from part i) of Theorem 3.9 is satisfied, too, for an arbitrary open, relatively compact, exhaustion $\left(X_{n}\right)_{n \in \mathbb{N}}$ of $X$. Hence, by Theorem 3.9, iii) implies i) in part a).

Mutatis mutandis, part b) of the theorem is again proved by applying Theorem 3.11 instead of Theorem 3.9 .

Theorem 5.3. Let $\omega$ be a weight function satisfying $\left(\alpha_{0}\right)$. Moreover, let $X \subseteq \mathbb{R}^{d}$ be open, $w \in \mathscr{E}_{\{\omega\}}(X)$, and $\psi: X \rightarrow X$ be smooth such that $\psi_{j} \in \mathscr{E}_{\{\omega\}}(X)$ for all $1 \leq j \leq d$.

a) The following are equivalent.

i) $C_{w, \psi}$ is weakly mixing on $\mathscr{E}\{\omega\}(X)$.

ii) $C_{w, \psi}$ is transitive on $\mathscr{E}_{\{\omega\}}(X)$.

iii) $w$ has no zeros, $\psi$ is injective, run-away, and detJ $\psi(x) \neq 0$ for all $x \in X$.

b) The following are equivalent.

i) $C_{w, \psi}$ is mixing on $\mathscr{E}_{\{\omega\}}(X)$.

ii) $w$ has no zeros, $\psi$ is injective, strong run-away, and $\operatorname{det} J \psi(x) \neq 0$ for all $x \in X$.

Proof. We first prove part a) of the theorem. Clearly, i) implies ii) and since polynomials are contained in $\mathscr{E}\{\omega\}\left(\mathbb{R}^{d}\right)$ the latter space is dense in $C\left(\mathbb{R}^{d}\right)$ so that by Theorem [3.9, iii) follows from ii). If iii) is satisfied, it follows as in the proof of Theorem [5.2 from [RaSc2, Theorem 3], and Remark [3.10 iii) that condition a) in part i) of Theorem 3.9 is fulfilled. Condition b3) of part i) in Theorem 3.9] is satisfied for any open, relatively compact exhaustion $\left(X_{n}\right)_{n \in \mathbb{N}}$ because by $\mathrm{HeMe}$, Proposition 3.2] $\mathscr{H}\left(\mathbb{C}^{d}\right)$ is dense in $\mathscr{E}_{\{\omega\}}(Y)$ for every open subset $Y$ of $\mathbb{R}^{d}$. From the run-away property and the injectivity of $\psi$ together with $\operatorname{det} J(x) \neq 0$ for all $x \in X$ it follows that conditions b1) and b2) from part i) of Theorem 3.9 are satisfied, too, so that i) follows.

The proof of part b) is once more a straight forward modification of the proof of part a) involving Theorem 3.11

Since for the weight function $\omega(t)=t$ the spaces $\mathscr{E}_{\{\omega\}}(X)$ and $\mathscr{A}(X)$ coincide as locally convex spaces it follows that Corollary 4.3 is a special case of Theorem 5.3 .

\section{KERNELS OF ELLIPTIC DIFFERENTIAL OPERATORS}

In this section we apply the results from section 3 to weighted composition operators defined on kernels of elliptic partial differential operators. The special case of the Cauchy-Riemann operator will give the space of holomorphic functions of a single variable equipped with the compact-open topology. In this context dynamical properties of (even a sequence of) unweighted composition operators have been studied by Bernal-González, Montes-Rodríguez BeMo, resp. GroßeErdmann, Mortini GEMo. For dynamical properties of weighted composition operators on the Fréchet space of holomorphic functions see also the articles YoRe] and $[\mathrm{Bes}]$.

The special case of the Laplace operator gives the space of harmonic functions endowed with the compact-open topology where dynamical properties of special unweighted composition operators have been studied for example in $\mathrm{Dz}, \mathrm{Ar}$. The results in this section complement those from [CaMu, $\mathrm{KaNi}$, and $\mathrm{KaNiRe}$ where hypercyclicity of special unweighted composition operators on spaces of zero solutions to linear partial differential equations with constant coefficients is considered.

As explained in example $2.3 \mathrm{v}$ ), for a non-constant polynomial with complex coefficients in $d \geq 2$ variables $P \in \mathbb{C}\left[X_{1}, \ldots, X_{d}\right]$ and an open subset $X \subseteq \mathbb{R}^{d}$ we 
define

$$
C_{P}^{\infty}(X):=\left\{u \in C^{\infty}(X) ; P(\partial) u=0 \text { in } X\right\},
$$

where for $P(\xi)=\sum_{|\alpha| \leq m} a_{\alpha} \xi^{\alpha}$ with $a_{\alpha_{0}} \neq 0$ for some multiindex $\alpha_{0} \in \mathbb{N}_{0}^{d}$ with $\left|\alpha_{0}\right|\left(=\alpha_{1}+\ldots+\alpha_{d}\right)=m$ we define

$$
\forall u \in C^{\infty}(X), x \in X: P(\partial) u(x)=\sum_{|\alpha| \leq m} a_{\alpha} \partial^{\alpha} u(x) .
$$

As a closed subspace of the separable nuclear Fréchet space $C^{\infty}(X)$ the space $C_{P}^{\infty}(X)$ is then again a separable nuclear Fréchet space. For hypoelliptic polynomials $P$ - by definition - for every open $X \subseteq \mathbb{R}^{d}$ the spaces $C_{P}^{\infty}(X)$ and

$$
\mathscr{D}_{P}^{\prime}(X):=\left\{u \in \mathscr{D}^{\prime}(X) ; P(\partial) u=0 \text { in } X\right\}
$$

coincide (that is, every distribution $u$ on $X$ which satisfies $P(\partial) u=0$ in $X$ is already a smooth function). By a result of Malgrange (see e.g. [Tr, Theorem 52.1]) the spaces $C_{P}^{\infty}(X)$ and $\mathscr{D}_{P}^{\prime}(X)$ also coincide as locally convex spaces when the latter is endowed with the relative topology inherited from $\mathscr{D}^{\prime}(X)$ equipped with the strong dual topology as the topological dual of $\mathscr{D}(X)$. This implies in particular, that for hypoelliptic polynomials the compact-open topology on $C_{P}^{\infty}(X)$ and the relative topology inherited from $C^{\infty}(X)$ coincide. Therefore, for hypoelliptic polynomials $P$ the space $C_{P}^{\infty}(X)$ endowed with the compact-open topology is a separable (nuclear) Fréchet space for every open $X \subseteq \mathbb{R}^{d}$.

As already mentioned in example $2.3 \mathrm{v}), C_{P}^{\infty}$ defines a sheaf on $\mathbb{R}^{d}$ which satisfies $(\mathscr{F} 1)$ and $(\mathscr{F} 2)$ but generally $(\mathscr{F} 3)$ need not hold. However, the next proposition shows that for hypoelliptic polynomials $P$ both $(\mathscr{F} 3)$ and $(\mathscr{F} 4)$ hold for $C_{P}^{\infty}$.

Proposition 6.1. Let $d \geq 2$ and let $P \in \mathbb{C}\left[X_{1}, \ldots, X_{d}\right]$ be hypoelliptic. The sheaf $C_{P}^{\infty}$ satisfies both $(\mathscr{F} 3)$ and $(\mathscr{F} 4)$.

Proof. Fix $x, y \in \mathbb{R}^{d}$ with $x \neq y$. By renumbering the coordinates we can assume without loss of generality that $x_{d}-y_{d} \neq 0$. For $\xi^{\prime} \in \mathbb{R}^{d-1}$ we denote by $\lambda_{1}\left(\xi^{\prime}\right), \ldots, \lambda_{l\left(\xi^{\prime}\right)}\left(\xi^{\prime}\right) \in \mathbb{C}$ the pairwise distinct roots of the polynomial

$$
\mathbb{C} \rightarrow \mathbb{C}, z \mapsto P\left(\xi^{\prime}, z\right),
$$

ordered in such a way that $\left(\operatorname{Im} \lambda_{j}\left(\xi^{\prime}\right)\right)_{1 \leq j \leq l\left(\xi^{\prime}\right)}$ is increasing and $\operatorname{Re} \lambda_{j}\left(\xi^{\prime}\right)<\operatorname{Re} \lambda_{j+1}\left(\xi^{\prime}\right)$ whenever $\operatorname{Im} \lambda_{j}\left(\xi^{\prime}\right)=\operatorname{Im} \lambda_{j+1}\left(\xi^{\prime}\right)$.

Then the mapping

$$
\operatorname{Im} \lambda_{1}: \mathbb{R}^{d-1} \rightarrow \mathbb{R}, \xi^{\prime} \mapsto \operatorname{Im} \lambda_{1}\left(\xi^{\prime}\right)
$$

is continuous. Indeed, fix $\xi_{0}^{\prime} \in \mathbb{R}^{d-1}$ and let $m_{j}$ be the multiplicities of the $\lambda_{j}\left(\xi_{0}^{\prime}\right)$. Let $\varepsilon>0$. Without loss of generality we assume that $\varepsilon$ is so small that for every $j$ we have $\operatorname{Im} \lambda_{j}\left(\xi_{0}^{\prime}\right)+\varepsilon<\operatorname{Im} \lambda_{j+1}\left(\xi_{0}^{\prime}\right)-\varepsilon$ if $\operatorname{Im} \lambda_{j}\left(\xi_{0}^{\prime}\right)<\operatorname{Im} \lambda_{j+1}\left(\xi_{0}^{\prime}\right)$ and $\operatorname{Re} \lambda_{j}\left(\xi_{0}^{\prime}\right)+\varepsilon<$ $\operatorname{Re} \lambda_{j+1}\left(\xi_{0}^{\prime}\right)-\varepsilon$ if $\operatorname{Im} \lambda_{j}\left(\xi_{0}^{\prime}\right)=\operatorname{Im} \lambda_{j+1}\left(\xi_{0}^{\prime}\right)$. Then $B\left(\lambda_{j}\left(\xi_{0}^{\prime}\right), \varepsilon\right) \cap B\left(\lambda_{k}\left(\xi_{0}^{\prime}\right), \varepsilon\right)=\emptyset$ for every $1 \leq j, k \leq l\left(\xi_{0}^{\prime}\right), j \neq k$. For any $1 \leq j \leq l\left(\xi_{0}^{\prime}\right)$ we have by Taylor's Theorem for every $\xi^{\prime} \in \mathbb{R}^{d-1}$ with $\left|\xi^{\prime}-\xi_{0}^{\prime}\right|<1$

$$
\begin{aligned}
\forall z \in \mathbb{C},\left|z-\lambda_{j}\left(\xi_{0}^{\prime}\right)\right| & =\varepsilon:\left|P\left(\xi^{\prime}, z\right)-P\left(\xi_{0}^{\prime}, z\right)\right| \\
& =\left|\sum_{\alpha \neq 0} P^{(\alpha)}\left(\left(\xi_{0}^{\prime}, 0\right)+z e_{d}\right) \frac{\left(\xi^{\prime}-\xi_{0}^{\prime}, 0\right)^{\alpha}}{\alpha !}\right| \\
& \leq \sum_{\alpha \neq 0}\left|P^{(\alpha)}\left(\left(\xi_{0}^{\prime}, 0\right)+z e_{d}\right)\right| \frac{\left|\xi^{\prime}-\xi_{0}^{\prime}\right|^{|\alpha|}}{\alpha !} \\
& \leq\left|\xi^{\prime}-\xi_{0}^{\prime}\right| \sup _{\zeta \in \mathbb{C},\left|\zeta-\lambda_{j}\left(\xi_{0}^{\prime}\right)\right|=\varepsilon} \sum_{\alpha \neq 0}\left|P^{(\alpha)}\left(\left(\xi_{0}^{\prime}, 0\right)+\zeta e_{d}\right)\right| \frac{1}{\alpha !}
\end{aligned}
$$


Thus, if $\left|\xi^{\prime}-\xi_{0}^{\prime}\right|$ is sufficiently small the right hand side of the above inequality is less than

$$
\inf \left\{\left|P\left(\xi_{0}^{\prime}, \zeta\right)\right| ; \zeta \in \mathbb{C},\left|\zeta-\lambda_{j}\left(\xi_{0}^{\prime}\right)\right|=\varepsilon\right\}\left(\leq\left|P\left(\xi_{0}^{\prime}, z\right)\right|\left(\left|z-\lambda_{j}\left(\xi_{0}^{\prime}\right)\right|=\varepsilon\right)\right)
$$

for any $j$. Hence it follows from Rouché's Theorem for $\xi^{\prime}$ sufficiently close to $\xi_{0}^{\prime}$, say $\left|\xi^{\prime}-\xi_{0}^{\prime}\right|<\delta$, that $P\left(\xi^{\prime}, \cdot\right)$ has exactly $m_{j} \operatorname{roots} z_{j, 1}^{\xi^{\prime}}, \ldots z_{j, m_{j}}^{\xi^{\prime}}$ in $B\left(\lambda_{j}\left(\xi_{0}^{\prime}\right), \varepsilon\right)$ for each $1 \leq j \leq l\left(\xi_{0}^{\prime}\right)$.

Now set $k:=\max \left\{1 \leq j \leq l\left(\xi_{0}^{\prime}\right) ; \operatorname{Im} \lambda_{1}\left(\xi_{0}^{\prime}\right)=\operatorname{Im} \lambda_{j}\left(\xi_{0}^{\prime}\right)\right\}$. Then for any $1 \leq j \leq$ $k, 1 \leq r \leq m_{j}$ we have

$$
\left|\operatorname{Im} z_{j, r}^{\xi^{\prime}}-\operatorname{Im} \lambda_{j}\left(\xi_{0}^{\prime}\right)\right|<\varepsilon
$$

if $\left|\xi^{\prime}-\xi_{0}^{\prime}\right|<\delta$ and according to our choice of $\varepsilon$ we have

$$
\begin{aligned}
& \max \left\{\operatorname{Im} z_{j, r}^{\xi^{\prime}} ; 1 \leq j \leq k, 1 \leq r \leq m_{j}\right\} \\
& <\min \left\{\operatorname{Im} z_{j, r}^{\xi^{\prime}} ; k+1 \leq j \leq k, 1 \leq r \leq m_{j}\right\} .
\end{aligned}
$$

Therefore $\operatorname{Im} \lambda_{1}\left(\xi^{\prime}\right), \ldots, \operatorname{Im} \lambda_{m_{1}}\left(\xi^{\prime}\right), \operatorname{Im} \lambda_{m_{1}+1}\left(\xi^{\prime}\right), \ldots, \operatorname{Im} \lambda_{m_{k}}\left(\xi^{\prime}\right)$ all belong to

$$
\left(\operatorname{Im} \lambda_{1}\left(\xi_{0}^{\prime}\right)-\varepsilon, \operatorname{Im} \lambda_{1}\left(\xi_{0}^{\prime}\right)+\varepsilon\right)=\ldots=\left(\operatorname{Im} \lambda_{k}\left(\xi_{0}^{\prime}\right)-\varepsilon, \operatorname{Im} \lambda_{k}\left(\xi_{0}^{\prime}\right)+\varepsilon\right)
$$

so that for all $1 \leq j \leq m_{k}$ we have $\left|\operatorname{Im} \lambda_{j}\left(\xi^{\prime}\right)-\operatorname{Im} \lambda_{1}\left(\xi_{0}^{\prime}\right)\right|<\varepsilon$ when $\left|\xi^{\prime}-\xi_{0}^{\prime}\right|<\delta$. In particular, $\left|\lambda_{1}\left(\xi^{\prime}\right)-\lambda_{1}\left(\xi_{0}^{\prime}\right)\right|<\varepsilon$ whenever $\left|\xi^{\prime}-\xi_{0}^{\prime}\right|<\delta$ which gives the continuity of $\operatorname{Im} \lambda_{1}$.

Denoting $V(P):=\left\{\zeta \in \mathbb{C}^{d} ; P(\zeta)=0\right\}$ it follows that $\left(\xi^{\prime}, \lambda_{1}\left(\xi^{\prime}\right)\right) \in V(P)$ for each $\xi^{\prime} \in \mathbb{R}^{d-1}$ and therefore

$$
\forall \xi^{\prime} \in \mathbb{R}^{d-1}: \operatorname{dist}\left(\left(\xi^{\prime}, \operatorname{Re} \lambda_{1}\left(\xi^{\prime}\right)\right), V(P)\right) \leq\left|\operatorname{Im} \lambda_{1}\left(\xi^{\prime}\right)\right| .
$$

Since $P$ is hypoelliptic we have

$$
\lim _{x \in \mathbb{R}^{d},|x| \rightarrow \infty} \operatorname{dist}(x, V(P))=\infty
$$

(see [Hö1, Theorem 11.1.3]) which combined with (6) yields

$$
\lim _{\left|\xi^{\prime}\right| \rightarrow \infty}\left|\operatorname{Im} \lambda_{1}\left(\xi^{\prime}\right)\right|=\infty
$$

This implies in particular that there are $\xi^{\prime}, \eta^{\prime} \in \mathbb{R}^{d-1}$ for which

$$
\begin{aligned}
& \left\langle\operatorname{Im}\left(\left(\xi^{\prime}, \lambda_{1}\left(\xi^{\prime}\right)\right)-\left(\eta^{\prime}, \lambda_{1}\left(\eta^{\prime}\right)\right)\right), x-y\right\rangle=\operatorname{Im}\left(\lambda_{1}\left(\xi^{\prime}\right)-\lambda_{1}\left(\eta^{\prime}\right)\right)\left(x_{d}-y_{d}\right) \\
& \notin\{2 \pi k ; k \in \mathbb{Z}\} .
\end{aligned}
$$

Thus, there are $\zeta_{1}, \zeta_{2} \in V(P)$ such that $\left\langle\operatorname{Im}\left(\zeta_{1}-\zeta_{2}\right), x-y\right\rangle$ is not an integer multiple of $2 \pi$. Setting $\langle\eta, v\rangle=\sum_{j=1}^{d} \eta_{j} v_{j}$ for $\eta, v \in \mathbb{C}^{d}$, the function

$$
g: \mathbb{R}^{d} \rightarrow \mathbb{C}, g(w):=\exp \left(\left\langle\zeta_{1}, x\right\rangle+\left\langle\zeta_{2}, w\right\rangle\right)-\exp \left(\left\langle\zeta_{2}, x\right\rangle+\left\langle\zeta_{1}, w\right\rangle\right)
$$

satisfies $g \in C_{P}^{\infty}\left(\mathbb{R}^{d}\right), g(x)=0$, and

$$
g(y)=\exp \left(\left\langle\zeta_{1}, y\right\rangle+\left\langle\zeta_{2}, x\right\rangle\right)\left(\exp \left(\left\langle\zeta_{1}-\zeta_{2}, x-y\right\rangle\right)-1\right) \neq 0
$$

which implies the existence of $f \in C_{P}^{\infty}\left(\mathbb{R}^{d}\right)$ with $f(x)=0$ and $f(y)=1$. Hence, $(\mathscr{F} 3)$ is satisfied.

To verify $(\mathscr{F} 4)$, let $X \subseteq \mathbb{R}^{d}$ be open. We first observe that $-\partial_{j} \delta_{x}, 1 \leq j \leq d$, is a continuous linear functional on $C_{P}^{\infty}(X)$. Now, let $h \in \mathbb{R}^{d} \backslash\{0\}$ and $\lambda \in \mathbb{K}$. By renumbering the coordinates if necessary we may assume that $h_{d} \neq 0$. By (77) it follows

$$
\exists \zeta \in V(P):\langle h, \operatorname{Im} \zeta\rangle-\operatorname{Im} \lambda \neq 0 .
$$


Because $e_{\zeta} \in C_{P}^{\infty}(X)$, where $e_{\zeta}(x):=\exp (\langle\zeta, x\rangle)$,

$$
\sum_{j=1}^{d} h_{j} \partial_{j} e_{\zeta}(x)-\lambda e_{\zeta}(x)=(\langle h, \operatorname{Re} \zeta\rangle-\operatorname{Re} \lambda+i(\langle h, \operatorname{Im} \zeta\rangle-\operatorname{Im} \lambda)) e_{\zeta}(x)
$$

where the factor

$$
(\langle h, \operatorname{Re} \zeta\rangle-\operatorname{Re} \lambda+i(\langle h, \operatorname{Im} \zeta\rangle-\operatorname{Im} \lambda))
$$

does not vanish by (8). Therefore the continuous linear functional

$$
u \mapsto \sum_{j=1}^{d} h_{j} \partial_{j} u-\lambda u
$$

on $C_{P}^{\infty}(X)$ does not vanish identically so that $(\mathscr{F} 4)$ is fulfilled.

For us, elliptic polynomials will be of particular interest. Recall that a polynomial $P \in \mathbb{C}\left[X_{1}, \ldots, X_{d}\right], P(\xi)=\sum_{|\alpha| \leq m} a_{\alpha} \xi^{\alpha}$ is called elliptic if

$$
\forall \xi \in \mathbb{R}^{d} \backslash\{0\} ; P_{m}(\xi) \neq 0,
$$

where $P_{m}(\xi)=\sum_{|\alpha|=m} a_{\alpha} \xi^{\alpha}$ denotes the principal part of $P$. As is well-known, elliptic polynomials are hypoelliptic (see e.g. [Hö1, Theorem 11.1.10]). In particular, identifying $\mathbb{C}$ as usual with $\mathbb{R}^{2}$, and choosing for $P \in \mathbb{C}\left[X_{1}, X_{2}\right]$ the polynomial $P\left(\xi_{1}, \xi_{2}\right)=\frac{1}{2}\left(\xi_{1}+i \xi_{2}\right)$ gives the Cauchy-Riemann operator $\partial_{\bar{z}}$ and we have that $C_{P}^{\infty}(X)=\mathscr{H}(X)$ holds as locally convex spaces for any open $X \subseteq \mathbb{C}$ so that the sheaf of holomorphic functions (equipped with the compact-open topology) on open subsets of $\mathbb{C}$ is a special case.

Arguably the most prominent elliptic differential operator, apart from the CauchyRiemann operator is the Laplace operator. Thus, the sheaf of harmonic functions (equipped with the compact-open topology) on open subsets of $\mathbb{R}^{d}$ is also a special case of the sheaves $C_{P}^{\infty}$.

We are now going to characterize when for an elliptic polynomial $P$ and an open $X \subseteq \mathbb{R}^{d}$ a well-defined weighted composition operator $C_{w, \psi}$ on $C_{P}^{\infty}(X)$ is weakly mixing. As follows in particular from the results obtained in GEMo an unweighted composition operator cannot be hypercyclic on $\mathscr{H}(X)$ if $X$ is a finitely connected but not simply connected domain. Thus, the special case of the Cauchy-Riemann operator shows that topological properties of $X$ have to be taken into account.

Theorem 6.2. Let $P$ be an elliptic polynomial and let $X \subseteq \mathbb{R}^{d}$ be open and homeomorphic to $\mathbb{R}^{d}$. Moreover, let $w: X \rightarrow \mathbb{C}$ and $\psi: X \rightarrow X$ be smooth such that $C_{w, \psi}$ is well-defined on $C_{P}^{\infty}(X)$ and acts locally on $C_{P}^{\infty}(X)$.

a) The following are equivalent.

i) $C_{w, \psi}$ is weakly mixing on $C_{P}^{\infty}(X)$.

ii) $C_{w, \psi}$ has dense range, $w$ has no zeros, and $\psi$ is injective and run-away.

iii) $w$ has no zeros, $\psi$ is injective and run-away, and for each $m \in \mathbb{N}_{0}$ and every open, relatively compact $Y \subseteq \psi^{m}(X)$ it holds

$r_{X}^{\left(\psi^{m}\right)^{-1}(Y)}\left(C_{P}^{\infty}(X)\right) \subseteq \overline{\left(C_{w, \psi,\left(\psi^{m-1}\right)^{-1}(Y)} \circ \ldots \circ C_{w, \psi, Y}\right)\left(C_{P}^{\infty}(Y)\right)}$,

where the closure is taken in $C_{P}^{\infty}\left(\left(\psi^{m}\right)^{-1}(Y)\right)$.

Moreover, $\operatorname{det} J \psi(x) \neq 0$ for all $x \in X$ can be added to ii) and iii). If additionally $|w(x)| \leq 1$ for all $x \in X$ then the above are equivalent to

iv) $C_{w, \psi}$ is hypercyclic on $C_{P}^{\infty}(X)$.

b) The following are equivalent.

i) $C_{w, \psi}$ is mixing on $C_{P}^{\infty}(X)$. 
ii) $C_{w, \psi}$ has dense range, $w$ has no zeros, and $\psi$ is injective and strong run-away.

iii) $w$ has no zeros, $\psi$ is injective and strong run-away, and for each $m \in$ $\mathbb{N}_{0}$ and every open, relatively compact $Y \subseteq \psi^{m}(X)$ it holds

$r_{X}^{\left(\psi^{m}\right)^{-1}(Y)}\left(C_{P}^{\infty}(X)\right) \subseteq \overline{\left(C_{w, \psi,\left(\psi^{m-1}\right)^{-1}(Y)} \circ \ldots \circ C_{w, \psi, Y}\right)\left(C_{P}^{\infty}(Y)\right)}$,

where the closure is taken in $C_{P}^{\infty}\left(\left(\psi^{m}\right)^{-1}(Y)\right)$.

Moreover, $\operatorname{det} J \psi(x) \neq 0$ for all $x \in X$ can be added to ii) and iii).

For the proof of Theorem 6.2 some preparations have to be made. Before providing these let us mention that in case of $\mathbb{C}=\mathbb{R}^{2}$ it follows from the Riemann Mapping Theorem that every simply connected, connected, open $X \subseteq \mathbb{C}$ different from $\mathbb{C}$ is in particular homeomorphic to the open unit disc in $\mathbb{C}$ which itself is homeomorphic to $\mathbb{C}$. Thus, in case of $d=2$ the topological hypothesis on $X$ in Theorem 6.2 means precisely that $X$ is a simply connected domain.

In order to prove Theorem 6.2 we need the following version of the celebrated Jordan-Brouwer Separation Theorem that can be found in May, Satz 5.23]. Since this reference is written in German and since we could not find a different reference we include a proof here - which is different from the one presented in [May - for the reader's convenience.

Theorem 6.3. (Version of Jordan-Brouwer Separation Theorem) Let $K_{1}$ and $K_{2}$ be homeomorphic compact subsets of $\mathbb{R}^{d}$. Then $\mathbb{R}^{d} \backslash K_{1}$ and $\mathbb{R}^{d} \backslash K_{2}$ have the same number of connected components.

Proof. For a topological space $X$ we denote as usual the $n$-th reduced homology group, respectively cohomology group, with coefficients in $\mathbb{Z}$ by $\tilde{H}_{n}(X, \mathbb{Z})$ respectively $\tilde{H}^{n}(X, \mathbb{Z})$. Moreover, let $S^{d}$ be the unit sphere in $\mathbb{R}^{d+1}, N:=(1,0, \ldots, 0) \in$ $S^{d}$ be the "north pole" and let $\phi: \mathbb{R}^{d} \rightarrow S^{d} \backslash\{N\}$ be a homeomorphism. Since $K_{1}$ and $K_{2}$ are homeomorphic it follows that the compact subsets $\{N\} \dot{\cup} \phi\left(K_{1}\right)$ and $\{N\} \dot{\cup} \phi\left(K_{2}\right)$ of $S^{d}$ are homeomorphic. Thus, the groups $\tilde{H}^{d-1}\left(\{N\} \dot{\cup} \phi\left(K_{1}\right), \mathbb{Z}\right)$ and $\tilde{H}^{d-1}\left(\{N\} \cup \dot{\varphi} \phi\left(K_{2}\right), \mathbb{Z}\right)$ are isomorphic. Using Alexander Duality (see [Ha, Theorem $3.44])$ it follows that the groups $\tilde{H}_{0}\left(S^{d} \backslash\left(\{N\} \dot{\cup} \phi\left(K_{1}\right)\right), \mathbb{Z}\right)$ and $\tilde{H}_{0}\left(S^{d} \backslash\left(\{N\} \dot{\cup} \phi\left(K_{2}\right)\right), \mathbb{Z}\right)$ are isomorphic and therefore, the same is true for the groups $\tilde{H}_{0}\left(\mathbb{R}^{d} \backslash K_{1}, \mathbb{Z}\right)$ and $\tilde{H}_{0}\left(\mathbb{R}^{d} \backslash K_{2}, \mathbb{Z}\right)$. Hence, the groups $\tilde{H}_{0}\left(\mathbb{R}^{d} \backslash K_{1}, \mathbb{Z}\right) \oplus \mathbb{Z}$ and $\tilde{H}_{0}\left(\mathbb{R}^{d} \backslash K_{2}, \mathbb{Z}\right) \oplus \mathbb{Z}$ are isomorphic as well. Since for any topological space $X$ the groups $\tilde{H}_{0}(X, \mathbb{Z}) \oplus \mathbb{Z}$ and $H_{0}(X, \mathbb{Z})$, the homology group of degree zero of $X$ are isomorphic (see e.g. $\mathrm{Ha}$, page 110]) and since $H_{0}(X, \mathbb{Z})$ is isomorphic to $\oplus_{\alpha \in C(X)} \mathbb{Z}$, where $C(X)$ is the set of all pathwise connected components of $X$, it follows that $\mathbb{R}^{d} \backslash K_{1}$ and $\mathbb{R}^{d} \backslash K_{2}$ have the same number of pathwise connected components. Since $\mathbb{R}^{d} \backslash K_{j}$ are open in $\mathbb{R}^{d}$ and thus locally pathwise connected it follows that $\mathbb{R}^{d} \backslash K_{1}$ and $\mathbb{R}^{d} \backslash K_{2}$ have indeed the same number of connected components.

Proposition 6.4. Denoting for $x \in \mathbb{R}^{d}$ and $\varepsilon>0$ the open, resp. closed ball about $x$ with radius $\varepsilon$ by $B(x, \varepsilon)$ and $B[x, \varepsilon]$, respectively, for every continuous and injective $\psi: \mathbb{R}^{d} \rightarrow \mathbb{R}^{d}$ the following hold.

i) $\forall n \in \mathbb{N}: \overline{\mathbb{R}^{d} \backslash \psi(B[0, n])}=\mathbb{R}^{d} \backslash \psi(B(0, n)), \overline{\mathbb{R}^{d} \backslash B[0, n]}=\mathbb{R}^{d} \backslash B(0, n)$.

ii) If $d \geq 2$ then $\mathbb{R}^{d} \backslash \psi(B[0, n])$ and $\mathbb{R}^{d} \backslash \psi(B(0, n))$ are connected for every $n \in \mathbb{N}$.

iii) If $d \geq 2$ and $n \in \mathbb{N}$ then $\mathbb{R}^{d} \backslash(\psi(B(0, n)) \cup B(0, n))$ is connected whenever $\psi(B[0, n]) \cap B[0, n]=\emptyset$.

Proof. Denoting the interior of $A \subseteq \mathbb{R}^{d}$ by $\operatorname{int}(A)$ we have $\mathbb{R}^{d} \backslash \operatorname{int}(A)=\overline{\mathbb{R}^{d} \backslash A}$ for any $A \subseteq \mathbb{R}^{d}$. Thus, $\overline{\mathbb{R}^{d} \backslash \psi(B[0, n])}=\mathbb{R}^{d} \backslash \operatorname{int}(\psi(B[0, n]))$. Since $\psi$ is continuous and 
injective it follows from Brouwer's Invariance of Domain Theorem that $\psi(B(0, n))$ is open in $\mathbb{R}^{d}$. Thus, $\psi(B(0, n)) \subseteq \operatorname{int}(\psi(B[0, n]))$. On the other hand, for $x$ in the interior of $\psi(B[0, n])$ there is $\delta>0$

$$
\psi^{-1}(B(x, \delta)) \subseteq \psi^{-1}(\psi(B[0, n]))=B[0, n],
$$

where we used the injectivity of $\psi$. Since $\psi^{-1}(B(x, \delta))$ is open in $\mathbb{R}^{d}$, we conclude $\psi^{-1}(B(x, \delta)) \subseteq B(0, n)$. From

$$
B(x, \delta) \subseteq \psi(B[0, n]) \subseteq \psi\left(\mathbb{R}^{d}\right)
$$

and the injectivity of $\psi$ we get

$$
B(x, \delta)=\psi\left(\psi^{-1}(B(x, \delta))\right) \subseteq \psi(B(0, n)) .
$$

Since $x \in \operatorname{int}(\psi(B[0, n]))$ was chosen arbitrarily it follows $\operatorname{int}(\psi(B[0, n])) \subseteq \psi(B(0, n))$ so that $\operatorname{int}(\psi(B[0, n]))=\psi(B(0, n))$ which proves i).

In order to prove ii), we define for $n \in \mathbb{N}$

$$
\psi_{n}: B[0, n] \rightarrow \psi(B[0, n]), x \mapsto \psi(x)
$$

which is a continuous bijection, thus a homeomorphism due to the compactness of $B[0, n] . \mathbb{R}^{d} \backslash B[0, n]$ is connected because $d \geq 2$ so that by Theorem 6.3 the same is true for $\mathbb{R}^{d} \backslash \psi(B[0, n])$. Therefore, using i), it follows that

$$
\overline{\mathbb{R}^{d} \backslash \psi(B[0, n])}=\mathbb{R}^{d} \backslash \psi(B(0, n))
$$

is connected, too, which proves ii).

In order to show iii), we first observe that

$$
\overline{\mathbb{R}^{d} \backslash(\psi(B[0, n]) \cup B(0, n))}=\mathbb{R}^{d} \backslash \operatorname{int}(\psi(B[0, n]) \cup B(0, n)) .
$$

Clearly,

$$
\operatorname{int}(\psi(B[0, n]) \cup B(0, n)) \supseteq \operatorname{int}(\psi(B[0, n])) \cup B(0, n)
$$

and because $\psi(B[0, n])$ and $B[0, n]$ are disjoint closed sets we also have

$$
\operatorname{int}(\psi(B[0, n]) \cup B(0, n)) \subseteq \operatorname{int}(\psi(B[0, n])) \cup B(0, n)
$$

which combined with $\operatorname{int}(\psi(B[0, n]))=\psi(B(0, n))$ gives

$$
\overline{\mathbb{R}^{d} \backslash(\psi(B[0, n]) \cup B(0, n))}=\mathbb{R}^{d} \backslash(\psi(B(0, n)) \cup B(0, n)) .
$$

Because the closure of a connected set is connected, it suffices to show the connectedness of the set $\mathbb{R}^{d} \backslash(\psi(B[0, n]) \cup B(0, n))$. Let $x, y$ be in the complement of $\psi(B[0, n]) \cup B(0, n)$. By ii $), \mathbb{R}^{d} \backslash \psi(B[0, n])$ is connected. Because open, connected subsets of $\mathbb{R}^{d}$ are pathwise connected, there is a continuous $\gamma:[0,1] \rightarrow$ $\mathbb{R}^{d} \backslash \psi(B[0, n])$ with $\gamma(0)=x$ and $\gamma(1)=y$. Without loss of generality, we can assume that $\gamma([0,1])$ does not intersect $B(0, n)$ because otherwise let

$$
t_{0}:=\inf \{t \in[0,1] ; \gamma(t) \in B(0, n)\}, t_{1}:=\sup \{t \in[0,1] ; \gamma(t) \in B(0, n)\} .
$$

Then $0<t_{0} \leq t_{1}<1$ and $\gamma\left(t_{j}\right) \in \partial B(0, n), j=0,1$. Since $d \geq 2$ the set $\partial B(0, n)$ is pathwise connected so there is a continuous $\alpha:\left[t_{0}, t_{1}\right] \rightarrow \partial B(0, n)$ such that $\alpha\left(t_{j}\right)=\gamma\left(t_{j}\right), j=0,1$. Then

$$
\left.\tilde{\gamma}:[0,1] \rightarrow \mathbb{R}^{d} \backslash(\psi(B[0, n])) \cup B(0, n)\right), t \mapsto \begin{cases}\gamma(t), & t \notin\left[t_{0}, t_{1}\right] \\ \alpha(t), & t \in\left[t_{0}, t_{1}\right]\end{cases}
$$

is a well-defined continuous mapping with $\tilde{\gamma}(0)=x$ and $\tilde{\gamma}(1)=y$.

This shows that $\mathbb{R}^{d} \backslash(\psi(B[0, n]) \cup B(0, n))$ is pathwise connected, a fortiori connected which proves iii). 
Proposition 6.5. Let $d \geq 2$ and $X \subseteq \mathbb{R}^{d}$ be open and homeomorphic to $\mathbb{R}^{d}$ as well as $\psi: X \rightarrow X$ be continuous, injective, and run-away. Then there is a relatively compact-open exhaustion $\left(X_{n}\right)_{n \in \mathbb{N}}$ of $X$ such that

$$
\forall n \in \mathbb{N} \exists m \in \mathbb{N}: X_{n} \cap \psi^{m}\left(X_{n}\right)=\emptyset
$$

and if $m, n \in \mathbb{N}$ are such that $X_{n}$ and $\psi^{m}\left(X_{n}\right)$ are disjoint, if $X \backslash\left(X_{n} \cup \psi^{m}\left(X_{n}\right)\right)=$ $F \cup \dot{ } K$ where $F$ is (relatively) closed in $X$ and $K \subseteq X$ is compact, then $K=\emptyset$.

Proof. We first assume that $X=\mathbb{R}^{d}$. From the hypothesis it follows that for each $n \in \mathbb{N}$ there is $m \in \mathbb{N}$ such that $B(0, n)$ and $\psi^{m}(B(0, n))$ are disjoint. Applying Proposition 6.4 to $\psi^{m}$ we obtain that $\mathbb{R}^{d} \backslash\left(B(0, n) \cup \psi^{m}(B(0, n))\right)$ is connected. In particular, for every closed set $F \subseteq \mathbb{R}^{d}$ and each compact subset $K$ of $\mathbb{R}^{d} \backslash F$ we have

$$
\mathbb{R}^{d} \backslash\left(B(0, n) \cup \psi^{m}(B(0, n))\right)=F \dot{\cup} K \Rightarrow K=\emptyset .
$$

Thus, for $X=\mathbb{R}^{d}$ we can choose $X_{n}:=B(0, n), n \in \mathbb{N}$.

Now let $X \subseteq \mathbb{R}^{d}$ be an arbitrary open subset homeomorphic to $\mathbb{R}^{d}$ via $\Phi: X \rightarrow$ $\mathbb{R}^{d}$. Then $\Phi \circ \psi \circ \Phi^{-1}$ is a continuous, injective mapping on $\mathbb{R}^{d}$ with the run-away property. For $n \in \mathbb{N}$ let $X_{n}:=\Phi^{-1}(B(0, n))$ so that $\left(X_{n}\right)_{n \in \mathbb{N}}$ is an open, relatively compact exhaustion of $X$. Let $n, m \in \mathbb{N}$ be such that $X_{n}$ and $\psi^{m}\left(X_{n}\right)$ are disjoint and let $F \subseteq X$ be relatively closed and $K \subseteq X$ be compact such that

$$
F \dot{\cup} K=X \backslash\left(X_{n} \cup \psi^{m}\left(X_{n}\right)\right)=\Phi^{-1}\left(\mathbb{R}^{d} \backslash\left(B(0, n) \cup(\Phi \circ \psi \circ \Phi)^{m}(B(0, n))\right)\right) .
$$

Since $\Phi(F)$ is a closed subset of $\mathbb{R}^{d}$ and $\Phi(K)$ is compact it follows together with

$$
\Phi(F) \cup \dot{\cup} \Phi(K)=\mathbb{R}^{d} \backslash\left(B(0, n) \cup(\Phi \circ \psi \circ \Phi)^{m}(B(0, n))\right)
$$

and the case of $X=\mathbb{R}^{d}$ applied to $\Phi \circ \psi \circ \Phi^{-1}$ that $\Phi(K)=\emptyset$ hence $K=\emptyset$.

Proof of Theorem 6.2. Since $P$ is elliptic the sheaf $C_{P}^{\infty}$ satisfies $(\mathscr{F} 1)-(\mathscr{F} 4)$ by Proposition 6.1. We can therefore invoke Theorem 3.9 in order to prove part a). If i) holds, i.e. if $C_{w, \psi}$ is weakly mixing, $C_{w, \psi}$ has obviously dense range and the rest of ii) follows from Theorem 3.9. If ii) holds, it follows from Proposition 3.8 that iii) is true.

Next, if iii) holds, condition a) from part i) of Theorem 3.9 is fulfilled. Let $\left(X_{n}\right)_{n \in \mathbb{N}}$ be the open, relatively compact exhaustion of $X$ from Proposition 6.5. By the injectivity of $\psi$ it follows from Brouwer's Invariance of Domain Theorem that b1) of part i) from Theorem 3.9 is satisfied, while b2) is satisfied since $\psi$ is run-away. Fix $n \in \mathbb{N}$ and let $m \in \mathbb{N}$ be such that $X_{n}$ and $\psi^{m}\left(X_{n}\right)$ are disjoint. It follows from Proposition 6.5 that it is not possible to decompose $X \backslash\left(X_{n} \cup \psi^{m}\left(X_{n}\right)\right)$ into a relatively closed subset of $X$ and a non-empty compact subset of $K$ which are disjoint. Since $P$ is elliptic it follows from the Lax-Malgrange Theorem (see e.g. Hö1, Theorem 4.4.5 combined with the remark preceding Corollary 4.4.4 resp. with Theorem 8.6.1] or [Na, Theorem 3.10.7]) that

$$
\left\{u_{\mid X_{n} \cup \psi^{m}\left(X_{n}\right)} ; u \in C_{P}^{\infty}(X)\right\}
$$

is dense in $C_{P}^{\infty}\left(X_{n} \cup \psi^{m}\left(X_{n}\right)\right)$, i.e. that $r_{X}^{X_{n} \cup \psi^{m}\left(X_{n}\right)}$ has dense range. Thus, conditions a) and b) from part i) of Theorem 3.9 are satisfied so that by this theorem $C_{w, \psi}$ is weakly mixing. Thus i)-iii) are equivalent. If additionally $|w(x)| \leq 1$ and $C_{w, \psi}$ is hypercyclic it follows from Theorem 3.9 that iii) holds. Since trivially i) implies iv), a) is proved.

The proof of part b) is mutatis mutandis a repetition of the above arguments with the reference to Theorem 3.9 replaced by a reference to Theorem 3.11 . 
In the remainder of this section we are going to characterize the dynamics for weighted composition operators on eigenspaces of the Cauchy-Riemann operator and Laplace operator respectively, i.e. on $C_{P}^{\infty}$ for the polynomial in $d=2$ variables $P(\xi)=\frac{1}{2}\left(\xi_{1}+i \xi_{2}\right)-\lambda$, resp. in $d$ variables $P(\xi)=\sum_{j=1}^{d} \xi_{j}^{2}-\lambda$ where in both cases $\lambda \in \mathbb{C}$ is arbitrary. We begin our considerations for these special operators by determining explicitly the combinations of symbols and weights which yield well-defined weighted composition operators on $C_{P}^{\infty}(X)$.

\section{Proposition 6.6.}

a) Let $\lambda \in \mathbb{C}$ and let $d=2$ as well as $P(\xi)=\frac{1}{2}\left(\xi_{1}+i \xi_{2}\right)-\lambda$. For $X \subseteq \mathbb{R}^{2}=\mathbb{C}$ open, $w: X \rightarrow \mathbb{C}$ and $\psi: X \rightarrow X$ smooth functions the following are equivalent.

i) $C_{w, \psi}$ is well-defined on $C_{P}^{\infty}(X)$.

ii) $w \partial_{\bar{z}} \psi=0$ and $P(\partial) w=-\lambda w \partial_{\bar{z}} \bar{\psi}$.

Moreover, if $C_{w, \psi}$ is well-defined on $C_{P}^{\infty}(X)$ it follows that for every $Y \subseteq X$ open and $f \in C^{\infty}(Y)$ we have

$$
P(\partial)\left(C_{w, \psi}(f)\right)=\partial_{\bar{z}} \bar{\psi} C_{w, \psi}(P(\partial) f) .
$$

b) Let $\lambda \in \mathbb{C}$ and let $d \geq 2$ as well as $P(\xi)=\sum_{j=1}^{d} \xi_{j}^{2}-\lambda$. For $X \subseteq \mathbb{R}^{d}$ open, $w: X \rightarrow \mathbb{C}$ and $\psi: X \rightarrow X$ smooth functions the following are equivalent.

i) $C_{w, \psi}$ is well-defined on $C_{P}^{\infty}(X)$.

ii) For every $1 \leq j \neq k \leq d$ it holds $w\left|\nabla \psi_{j}\right|^{2}=w\left|\nabla \psi_{k}\right|^{2}$ as well as $w\left\langle\nabla \psi_{j}, \nabla \psi_{k}\right\rangle=0, w \Delta \psi_{j}+2\left\langle\nabla w, \nabla \psi_{j}\right\rangle=0$, and $P(\partial) w=-\lambda w\left|\nabla \psi_{1}\right|^{2}$. Moreover, if $C_{w, \psi}$ is well-defined on $C_{P}^{\infty}(X)$ it follows that for every $Y \subseteq X$ open and $f \in C^{\infty}(Y)$ we have

$$
P(\partial)\left(C_{w, \psi}(f)\right)=\left|\nabla \psi_{1}\right|^{2} C_{w, \psi}(P(\partial) f) .
$$

Proof. We use the notation $f(\psi)$ instead of $f \circ \psi$ in order to slightly simplify notation. In order to prove a) it is straightforward to verify that for every $f \in$ $C^{\infty}(X)$

$$
P(\partial)(w \cdot f(\psi))=(P(\partial) w) f(\psi)+w\left(\partial_{z} f\right)(\psi) \partial_{\bar{z}} \psi+w\left(\partial_{\bar{z}} f\right)(\psi) \partial_{\bar{z}} \bar{\psi} .
$$

Now assume that i) in part a) holds. Inserting $f=e_{\zeta}$ with $\zeta=(2 \lambda, 0) \in \mathbb{C}^{2}$ into equation (9) we obtain from $e_{\zeta} \in C_{P}^{\infty}(X)$ (recall that $e_{\zeta}(x)=\exp \left(\sum_{j=1}^{2} \zeta_{j} x_{j}\right)$ ) that

$$
0=\left(P(\partial) w+\lambda w\left(\partial_{\bar{z}} \psi+\partial_{\bar{z}} \bar{\psi}\right)\right) e_{\zeta}
$$

so that

$$
0=P(\partial) w+\lambda w\left(\partial_{\bar{z}} \psi+\partial_{\bar{z}} \bar{\psi}\right) .
$$

Likewise, we derive from equation (9) by inserting $f=e_{\eta}$ with $\eta=(0,-2 i \lambda) \in \mathbb{C}^{2}$ that

$$
0=P(\partial) w-\lambda w\left(\partial_{\bar{z}} \psi-\partial_{\bar{z}} \bar{\psi}\right) .
$$

Substracting equation (11) from equation (10) yields

$$
0=\lambda w \partial_{\bar{z}} \psi
$$

while adding equations (10) and (11) gives

$$
0=P(\partial) w+\lambda w \partial_{\bar{z}} \bar{\psi} .
$$

In case of $\lambda=0$ we evaluate equation (9) for $f(x)=x_{1}+i x_{2}$ which gives $w \partial_{\bar{z}} \psi=0$. In case of $\lambda \neq 0$ we have $w \partial_{\bar{z}} \psi=0$, too, by equation (12) showing one half of ii). 
Additionally, evaluating equation (9) for arbitrary $f \in C_{P}^{\infty}(X)$ gives

$$
\begin{aligned}
\forall f \in C_{P}^{\infty}(X): 0 & =(P(\partial) w) f(\psi)+w\left(\partial_{z} f\right)(\psi) \partial_{\bar{z}} \psi+w\left(\partial_{\bar{z}} f\right)(\psi) \partial_{\bar{z}} \bar{\psi} \\
& =(P(\partial) w) f(\psi)+\lambda w f(\psi) \partial_{\bar{z}} \bar{\psi} \\
& =\left(\partial_{\bar{z}} w-\left(1-\partial_{\bar{z}} \bar{\psi}\right) \lambda w\right) f(\psi) .
\end{aligned}
$$

Inserting $e_{\zeta}$ with $\zeta$ as above into this equation gives $\partial_{\bar{z}} w-\left(1-\partial_{\bar{z}} \bar{\psi}\right) \lambda w=0$ which proves that a) i) implies a) ii).

On the other hand, if a) ii) is satisfied, it follows from equation (9) that for every $f \in C_{P}^{\infty}(X)$ we have

$$
P(\partial)\left(C_{w, \psi}(f)\right)=\partial_{\bar{z}} \bar{\psi} C_{w, \psi}(P(\partial) f)
$$

which proves a) i).

To finish the proof of a), let $Y \subseteq X$ be open and assume that $C_{w, \psi}$ is well-defined on $C_{P}^{\infty}(X)$. Using a) ii) it is straightforward to derive - compare equation (9)

$$
\forall f \in C^{\infty}(Y): P(\partial)\left(C_{w, \psi}(f)\right)=\partial_{\bar{z}} \bar{\psi} C_{w, \psi}(P(\partial) f) .
$$

In order to prove b), we first notice that for $f \in C^{\infty}(X)$

$$
\begin{aligned}
P(\partial)(w \cdot f(\psi)) & =P(\partial) w \cdot f(\psi)+\sum_{l=1}^{d}\left(2\left\langle\nabla w, \nabla \psi_{l}\right\rangle+w \Delta \psi_{l}\right) \partial_{l} f(\psi) \\
& +w\left(\sum_{l=1}^{d} \sum_{m=1}^{d}\left(\partial_{l} \partial_{m} f\right)(\psi)\left\langle\nabla \psi_{l}, \nabla \psi_{m}\right\rangle\right) .
\end{aligned}
$$

We first show that b) i) implies b) ii). Inserting $f=e_{\zeta_{j}}, 1 \leq j \leq d$, with $\zeta_{j}=$ $\sqrt{\lambda}\left(\delta_{j, l}\right)_{1 \leq l \leq d}$ for any root $\sqrt{\lambda}$ of $\lambda$ it follows from $e_{\zeta_{j}} \in C_{P}^{\infty}(X)$ that

$$
0=\left(P(\partial) w+\sqrt{\lambda}\left(2\left\langle\nabla w, \nabla \psi_{j}\right\rangle+w \Delta \psi_{j}\right)+\lambda w\left|\nabla \psi_{j}\right|^{2}\right) e_{\zeta_{j}}
$$

so that for every $1 \leq j \leq d$

$$
\begin{aligned}
0 & =P(\partial) w+\sqrt{\lambda}\left(2\left\langle\nabla w, \nabla \psi_{j}\right\rangle+w \Delta \psi_{j}\right)+\lambda w\left|\nabla \psi_{j}\right|^{2} \\
& =\left(\Delta w-\left(1-\left|\nabla \psi_{j}\right|^{2}\right) \lambda w\right)+\sqrt{\lambda}\left(2\left\langle\nabla w, \nabla \psi_{j}\right\rangle+w \Delta \psi_{j}\right) .
\end{aligned}
$$

Analogously, inserting $f=e_{\tilde{\zeta}_{j}}, 1 \leq j \leq d$, with $\tilde{\zeta}_{j}=-\sqrt{\lambda}\left(\delta_{j, k}\right)_{1 \leq k \leq d}$ into equation (14) yields for $1 \leq j \leq d$

$$
0=\left(\Delta w-\left(1-\left|\nabla \psi_{j}\right|^{2}\right) \lambda w\right)-\sqrt{\lambda}\left(2\left\langle\nabla w, \nabla \psi_{j}\right\rangle+w \Delta \psi_{j}\right) .
$$

Adding equations (15) and (16) gives

$$
0=\Delta w-\left(1-\left|\nabla \psi_{j}\right|^{2}\right) \lambda w=P(\partial) w+\lambda\left|\nabla \psi_{j}\right|^{2} w
$$

for every $1 \leq j \leq d$ while substracting equation (16) from equation (15) gives

$$
\forall 1 \leq j \leq d: 0=2\left\langle\nabla w, \nabla \psi_{j}\right\rangle+w \Delta \psi_{j}
$$

for $\lambda \neq 0$. In case of $\lambda=0$ we have that $f(x)=x_{j} \in C_{P}^{\infty}(X)$ and plugging this $f$ into (14) shows that (18) is also valid for $\lambda=0$.

Next we insert $f=e_{\eta_{j, k}^{ \pm}}, 1 \leq j \neq k \leq d$, into equation (14), where for $\alpha \in$ $\mathbb{C} \backslash\{0,-\lambda\} \eta_{j, k}^{ \pm}=\sqrt{\lambda+\alpha}\left(\delta_{j, l}\right)_{1 \leq l \leq d} \pm i \sqrt{\alpha}\left(\delta_{k, l}\right)_{1 \leq l \leq d}$ resulting in

$$
\begin{aligned}
0 & =P(\partial) w+\sqrt{\lambda+\alpha}\left(2\left\langle\nabla w, \nabla \psi_{j}\right\rangle+w \Delta \psi_{j}\right) \\
& \pm i \sqrt{\alpha}\left(2\left\langle\nabla w, \nabla \psi_{k}\right\rangle+w \Delta \psi_{k}\right) \\
& +w\left((\lambda+\alpha)\left|\nabla \psi_{j}\right|^{2}-\alpha\left|\psi_{k}\right|^{2} \pm 2 i \sqrt{\alpha} \sqrt{\lambda+\alpha}\left\langle\nabla \psi_{j}, \nabla \psi_{k}\right\rangle\right) .
\end{aligned}
$$


Substracting from the version with "+" of the above equation the version with "-" yields

$$
0=2 i \sqrt{\alpha}\left(2\left\langle\nabla w, \nabla \psi_{k}\right\rangle+w \Delta \psi_{k}\right)+w 4 i \sqrt{\alpha} \sqrt{\lambda+\alpha}\left\langle\nabla \psi_{j}, \nabla \psi_{k}\right\rangle
$$

so taking into account equation (18) we derive

$$
\forall 1 \leq j \neq k \leq d: 0=w\left\langle\nabla \psi_{j}, \nabla \psi_{k}\right\rangle .
$$

Taking into account (18) and (20), equation (19) combined with (17) gives

$$
\begin{aligned}
0 & =\Delta w-\left(1-\left|\nabla \psi_{j}\right|^{2}\right) \lambda w+\alpha w\left(\left|\nabla \psi_{j}\right|^{2}-\left|\nabla \psi_{k}\right|^{2}\right) \\
& =\alpha w\left(\left|\nabla \psi_{j}\right|^{2}-\left|\nabla \psi_{k}\right|^{2}\right) .
\end{aligned}
$$

Since $\alpha \neq 0$, equation (21) together with equations (18) and (20) now give ii) in b).

On the other hand, if ii) of b) is satisfied, equation (14) simplifies to

$$
\begin{aligned}
\forall f \in C^{\infty}(X): P(\partial)(w \cdot f(\psi)) & =-\lambda w\left|\nabla \psi_{1}\right|^{2} \cdot f(\psi)+w\left|\nabla \psi_{1}\right|^{2}(\Delta f)(\psi) \\
& =\left|\nabla \psi_{1}\right|^{2} w(P(\partial) f)(\psi),
\end{aligned}
$$

in particular $C_{w, \psi}$ is well-defined on $C_{P}^{\infty}(X)$ proving b) i).

To finish the proof of b), let $Y \subseteq X$ be open and assume that $C_{w, \psi}$ is well-defined on $C_{P}^{\infty}(X)$. Using b) ii) it is straightforward to derive - compare equation (14)

$$
\forall f \in C^{\infty}(Y): P(\partial)\left(C_{w, \psi}(f)\right)=\left|\nabla \psi_{1}\right|^{2} C_{w, \psi}(P(\partial) f) .
$$

Corollary 6.7. Let $d \geq 2, \lambda \in \mathbb{C}$ and let $P(\xi)=\sum_{j=1}^{d} \xi_{j}^{2}-\lambda$. Moreover, let $X \subseteq \mathbb{R}^{d}$ be homeomorphic to $\mathbb{R}^{d}$ and assume that the smooth mappings $w: X \rightarrow \mathbb{C}$ and $\psi: X \rightarrow X$ are such that $C_{w, \psi}$ is well-defined on $C_{P}^{\infty}(X)$.

a) For $C_{w, \psi}$ the following are equivalent.

i) $C_{w, \psi}$ is weakly mixing on $C_{P}^{\infty}(X)$.

ii) $w$ has no zeros, $\psi$ is injective as well as run-away and satisfies $\operatorname{det} J \psi(x) \neq$ 0 for each $x \in X$.

If additionally $|w(x)| \leq 1$ for all $x \in X$ the above are equivalent to

iii) $C_{w, \psi}$ is hypercyclic on $C_{P}^{\infty}(X)$.

b) For $C_{w, \psi}$ the following are equivalent.

i) $C_{w, \psi}$ is mixing on $C_{P}^{\infty}(X)$.

ii) $w$ has no zeros, $\psi$ is injective as well as strong run-away and $\operatorname{det} J \psi(x) \neq$ 0 for each $x \in X$.

Proof. Because $C_{w, \psi}$ is well-defined on $C_{P}^{\infty}(X)$ it follows from Proposition 6.6 b) that for all $Y \subseteq X$ open we have

$$
\forall f \in C^{\infty}(Y): P(\partial)\left(C_{w, \psi}(f)\right)=\left|\nabla \psi_{1}\right|^{2} C_{w, \psi}(P(\partial) f)
$$

Clearly, (22) implies that $C_{w, \psi}$ acts locally on $C_{w, \psi}(X)$ so that by Theorem 6.2 we only have to show that a) ii) implies a) i) and that b) ii) implies b) i), respectively. This will be done once we have shown that under a) ii), respectively b) ii), it holds that for each $m \in \mathbb{N}_{0}$ and every open, relatively compact $Y \subseteq \psi^{m}(X)$ we have

$$
r_{X}^{\left(\psi^{m}\right)^{-1}(Y)}\left(C_{P}^{\infty}(X)\right) \subseteq \overline{\left(C_{w, \psi,\left(\psi^{m-1}\right)^{-1}(Y)} \circ \ldots \circ C_{w, \psi, Y}\right)\left(C_{P}^{\infty}(Y)\right)} .
$$

If a) ii), respectively b) ii), holds we have $\operatorname{det} J \psi(x) \neq 0$ for each $x \in X$ and we conclude that $\psi^{m}(X)$ is open and $\left(\psi^{m}\right)^{-1}: \psi^{m}(X) \rightarrow X$ is smooth for every $m \in \mathbb{N}$ as well as $\left|\nabla \psi_{1}(x)\right|^{2} \neq 0$ for all $x \in X$. Moreover, (22) implies for every open set $Y \subseteq \psi^{m}(X)$ and every $f \in C^{\infty}\left(\left(\psi^{m}\right)^{-1}(Y)\right)$ that

$$
P(\partial) f=\left|\nabla \psi_{1}\right|^{2 m} \prod_{j=0}^{m-1} w\left(\psi^{j}(\cdot)\right)\left(\left(P(\partial)\left[\left(\frac{f}{\prod_{j=0}^{m-1} w\left(\psi^{j}(\cdot)\right)}\right) \circ\left(\psi^{m}\right)^{-1}\right]\right) \circ \psi^{m}\right)
$$


which in turn yields

$$
P(\partial)\left(\left(\frac{f}{\prod_{j=0}^{m-1} w\left(\psi^{j}(\cdot)\right)}\right) \circ\left(\psi^{m}\right)^{-1}\right)=\left(\frac{P(\partial) f}{\left|\nabla \psi_{1}\right|^{2 m} \prod_{j=0}^{m-1} w\left(\psi^{j}(\cdot)\right)}\right) \circ\left(\psi^{m}\right)^{-1} .
$$

Therefore, for each $m \in \mathbb{N}$, for every open $Y \subseteq \psi^{m}(X)$, and every $f \in C_{P}^{\infty}\left(\left(\psi^{m}\right)^{-1}(Y)\right)$ it follows

$$
\left(\frac{f}{\prod_{j=0}^{m-1} w\left(\psi^{j}(\cdot)\right)}\right) \circ\left(\psi^{m}\right)^{-1} \in C_{P}^{\infty}(Y)
$$

so that by Remark 3.10 iii) we have

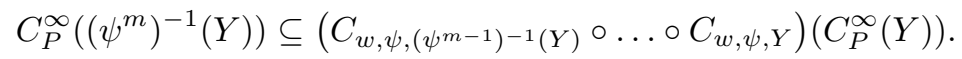

In particular,

$$
r_{X}^{\left(\psi^{m}\right)^{-1}(Y)}\left(C_{P}^{\infty}(X)\right) \subseteq \overline{\left(C_{w, \psi,\left(\psi^{m-1}\right)^{-1}(Y)} \circ \ldots \circ C_{w, \psi, Y}\right)\left(C_{P}^{\infty}(Y)\right)}
$$

which is all that had to be shown.

We close this section by applying Theorem 6.2 to characterize dynamics of weighted composition operators on eigenspaces of the Cauchy-Riemann operator. For the case $\lambda=0$ the equivalence of iii) and iv) is established in YoRe while the equivalence of iii) and i) for $\lambda=0$ was also considered in Bes, both without any restriction on the range of the weight $w$.

Corollary 6.8. Let $d=2, \lambda \in \mathbb{C}$ and let $P(\xi)=\frac{1}{2}\left(\xi_{1}+i \xi_{2}\right)-\lambda$. Moreover, let $X \subseteq \mathbb{R}^{2}$ be homeomorphic to $\mathbb{R}^{2}$ and assume that the smooth mappings $w: X \rightarrow \mathbb{C}$ and $\psi: X \rightarrow X$ are such that $C_{w, \psi}$ is well-defined on $C_{P}^{\infty}(X)$. Then, the following are equivalent.

i) $C_{w, \psi}$ is mixing on $C_{P}^{\infty}(X)$

ii) $C_{w, \psi}$ is weakly mixing on $C_{P}^{\infty}(X)$.

iii) $w$ has no zeros, $\psi$ is injective, holomorphic, and has no fixed point.

If additionally $|w(x)| \leq 1$ for all $x \in X$ the above are equivalent to

iv) $C_{w, \psi}$ is hypercyclic on $C_{P}^{\infty}(X)$.

Proof. We identify $\mathbb{R}^{2}$ with $\mathbb{C}$. Because $C_{w, \psi}$ is well-defined on $C_{P}^{\infty}(X)$ it follows from Proposition 6.6 b) that for all $Y \subseteq X$ open we have

$$
\forall f \in C^{\infty}(Y): P(\partial)\left(C_{w, \psi}(f)\right)=\partial_{\bar{z}} \bar{\psi} C_{w, \psi}(P(\partial) f)
$$

Equation (23) implies that $C_{w, \psi}$ acts locally on $C_{w, \psi}(X)$.

Clearly, i) implies ii) and by Theorem 6.2, if ii) holds, in particular, $w$ has no zeros, $\psi$ is injective and run-away. Especially, $\psi$ has no fixed point. Since $w$ has no zeros it follows from Proposition [6.6 a) ii) that $\psi$ is holomorphic so that ii) implies iii).

Next, if iii) holds, the composition operator $C_{\psi}$ is in particular a well-defined continuous linear operator on $\mathscr{H}(X)$, the holomorphic functions on $X$. Since $X$ is homeomorphic to $\mathbb{C}, X$ is a simply connected domain in $\mathbb{C}$ and because $\psi$ is injective and has no fixed point, it follows from [Bes, Proof of Theorem 3.1] that $\psi$ is strong run-away. From the injectivity of the holomorphic mapping $\psi$ we conclude that $0 \neq\left|\psi^{\prime}(z)\right|=\operatorname{det} J \psi(z)$ for all $z \in X$ (see e.g $[\mathbf{R u}$, Theorem 10.33]). In view of Theorem 6.2 we only have to show that for each $m \in \mathbb{N}_{0}$ and every open, relatively compact $Y \subseteq \psi^{m}(X)$ we have

$$
r_{X}^{\left(\psi^{m}\right)^{-1}(Y)}\left(C_{P}^{\infty}(X)\right) \subseteq \overline{\left(C_{w, \psi,\left(\psi^{m-1}\right)^{-1}(Y)} \circ \ldots \circ C_{w, \psi, Y}\right)\left(C_{P}^{\infty}(Y)\right)} .
$$

From $0 \neq \operatorname{det} J \psi(x)=\left|\partial_{z} \psi(x)\right|^{2}$ for all $x \in X$ together with $\partial_{\bar{z}} \bar{\psi}=\overline{\partial_{z} \psi}$ it follows that $\partial_{\bar{z}} \bar{\psi}$ has no zeros in $X$. Thus, equation (23) can be used as equation (22) in the proof of Corollary 6.7 to prove that i) holds. 
Finally, if $|w(x)| \leq 1$ for all $x \in X$ it follows from Theorem 6.2 that ii) and iv) are equivalent which completes the proof.

Motivated by the previous result we close this paper with two open problems. While the first one is concerned with the general abstract setting the second one aims at a more manageable characterization of hypercyclicity/mixing for weighted composition operators on eigenspaces of the Laplace operator.

Problem 6.9. 1. Are the additional assumptions in Proposition $3.4 v$ ) superfluous to prove that $\psi$ is run-away whenever $C_{w, \psi}$ is transitive? If this is the case, the additional assumption on $w$ (or the sheaf $\mathscr{F}$ ) in Theorem 3.9 can be removed so that i)-iv) in Theorem [6.2 are equivalent as well as i)-iii) in Corollary [6.7 a) and i)-iv) in Corollary [6.8, without the additional assumption on the range of the weight $w$.

2. Let $X \subseteq \mathbb{R}^{d}$ be homeomorphic to $\mathbb{R}^{d}, \lambda \in \mathbb{C}$, and $P(\xi)=\sum_{j=1}^{d} \xi_{j}^{2}-\lambda$. Characterize those $w \in C^{\infty}(X),|w| \leq 1$ and smooth $\psi: X \rightarrow X$ such that

i) $\forall 1 \leq j \neq k \leq d:\left|\nabla \psi_{j}\right|^{2}=\left|\nabla \psi_{k}\right|^{2}$ and $\left\langle\nabla \psi_{j}, \nabla \psi_{k}\right\rangle=0$,

ii) $\forall 1 \leq j \leq d: w \Delta \psi_{j}+2\left\langle\nabla w, \nabla \psi_{j}\right\rangle=0$ and $\Delta w-\lambda w=-\lambda\left|\nabla \psi_{1}\right|^{2}$,

iii) $\psi$ is run-away.

Are there hypercyclic weighted composition operators on $C_{P}^{\infty}(X)$ which are not mixing?

Acknowledgements. The author would like to thank J. Wengenroth for pointing out Alexander Duality, [Ha, Theorem 3.44], in connection with the version of the Jordan-Brouwer Separation Theorem which is used in this article. Moreover, the author is indebted to one of the anonymous referees for pointing out a gap in the proof of the previous version of Proposition $3.4 \mathrm{v}$ ).

\section{REFERENCES}

[AbZa] J. Abe, P. Zappa, Universal functions on complex general linear groups, J. Approx. Theory 100 (1999), no. 2, 221-232.

[Ar] D.H. Armitage, Harmonic monsters, J. Approx. Theory 123 (2003), no. 1, 110-116.

[BaMa] F. Bayart, É. Matheron, Hypercyclic operators failing the hypercyclicity criterion on classical Banach spaces, J. Funct. Anal. 250 (2007), no. 2, 426-441.

[BaMa2] F. Bayart, É. Matheron, Dynamics of linear operators, Cambridge Tracts in Mathematics, 179. Cambridge University Press, Cambridge, 2009.

[Ber] L. Bernal-González, Universal entire functions for affine endomorphisms of $\mathbb{C}^{N}$, J. Math. Anal. Appl. 305 (2005), no. 2, 690-697.

[BeMo] L. Bernal González, A. Montes-Rodríguez, Universal functions for composition operators, Complex Variables Theory Appl. 27 (1995), no. 1, 47-56.

[Bes] J. Bès, Dynamics of weighted composition operators, Complex Anal. Oper. Theory 8 (2014), no. 1, 159-176.

[Beu] A. Beurling, Quasi-analyticity and general distributions, Lectures 4 and 5, AMS Summer Institute, Stanford, 1961.

[Bi] G.D. Birkhoff, Démonstration d'un théorème élémentaire sur les fonctions entières, C.R. Acad. Sci. Paris 189 (1929), 473-475.

[Bj] G. Björck, Linear partial differential operators and generalized distributions, Ark. Mat. 6 (1966), 351-407.

[BoDo] J. Bonet, P. Domański, Hypercyclic composition operators on spaces of real analytic functions, Math. Proc. Camb. Phil. Soc. 153 (2012), no. 3, 489-503.

[BoMeMe] J. Bonet, R. Meise, S.N. Melikhov, A comparison of two different ways to define classes of ultradifferentiable functions, Bull. Belg. Math. Soc. Simon Stevin 14 (2007), no. 3, 425-444.

[BoSh] P.S. Bourdon, J.H. Shapiro, Cyclic phenomena for composition operators, Mem. Amer. Math. Soc. 125 (1997), no. 596.

[BrMeTa] R.W. Braun, R. Meise, B.A. Taylor, Ultradifferentiable functions and Fourier analysis, Results Math. 17 (1990), no. 3-4, 206-237. 
[Br] G.E. Bredon, Topology and Geometry, Graduate Texts in Mathematics 139, Springer, New York, 1993.

[CaMu] M.C. Calderón-Moreno, J. Müller, Universal holomorphic and harmonic functions with additional properties, Acta Math. Hungar. 105 (2004), no. 1-2, 1-15.

[DeVi] A. Debrouwere, J. Vindas, Solution to the first Cousin problem for vector-valued quasianalytic functions, Ann. Mat. Pura Appl. 196 (2017), no. 6, 1983-2003.

[DR] M. De la Rosa, C. Read, A hypercyclic operator whose direct sum $T \oplus T$ is not hypercyclic, J. Operator Theory 61 (2009), no. 2, 369-380.

[Dz] O.P. Dzagnidze, The universal harmonic function in the space $E_{n}$, Sakharth. SSR Mecn. Akad. Moambe 55 (1969), 41-44.

[En] R. Engelking, General Topology, Sigma Series in Pure Mathematics, 6. Heldermann Verlag, Berlin, 1989.

[FeGa] C. Fernández, A. Galbis, Superposition in classes of ultradifferentiable functions, Publ. Res. Inst. Math. Sci. 42 (2006), no. 2, 399-419.

[FrGr] K. Fritzsche, H. Grauert, From Holomorphic Functions to Complex Manifolds, Graduate Texts in Mathematics 213, Springer, New York, 2002.

[GaMo] E.A. Gallardo-Gutiérrez, A. Montes-Rodríguez, The role of the spectrum in the cyclic behavior of composition operators, Mem. Amer. Math. Soc. 167 (2004), no. 791.

[GEMo] K.G. Große-Erdmann, R. Mortini, Universal functions for composition operators with non-automorphic symbol, J. Math. Anal. 107 (2009), 355-376.

[GEPe] K.G. Große-Erdmann, A. Peris, Linear Chaos, Universitext, Springer, London, 2011.

[Ha] A. Hatcher, Algebraic Topology, Cambridge University Press, 2002.

[He] D.A. Herrero, Hypercyclic operators and chaos, J. Operator Theory 28 (1992), no. 1, 93-103.

[HeMe] T. Heinrich, R. Meise, A support theorem for analytic functionals, Math. Nachr. 280 (2007), no. 4, 364-387.

[Hö1] L. Hörmander, The Analysis of Linear Partial Differential Operators I and II, Classics in Mathematics, Springer, Berlin, 1983.

[Hö2] L. Hörmander, An Introduction to Complex Analysis in Several Variables, D. Van Nostrand Co., Inc., Princeton, N.J.Toronto, Ont.London, 1966.

[Ka] T. Kalmes, Hypercyclic, mixing, and chaotic $C_{0}$-semigroups induced by semiflows, Ergodic Theory Dynam. Systems 27 (2007), no. 5, 1599-1631.

[KaNi $\quad$ T. Kalmes, M. Niess, Universal zero-solutions of linear partial differential operators, Studia Math. 198 (2010), no. 1, 33-51.

[KaNiRe] T. Kalmes, M. Niess, T. Ransford, Examples of quantitative universal approximation, in: Complex analysis and potential theory, CRM Proc. Lecture Notes 55, Amer. Math. Soc., Providence, RI, 2012, 77-97.

[LS] F. Leon-Saavedra, Universal functions on the unit ball and the polydisk, in: Function spaces. Proceedings of the 3rd Conference held at Southern Illinois University, Edwardsville, IL, May 19-23, 1998, edited by K. Jarosz, Contemp. Math. 232, American Mathematical Society, Providence, RI, 1999, 233-238.

[Mar] A. Martineau, Sur la topologie des espaces de fonctions holomorphes, Math. Ann. 163 (1966), 62-88.

[May] K.H. Mayer Algebraische Topologie, Birkhäuser Verlag, Basel-Boston-Berlin, 1989.

[MeVo] R. Meise, D. Vogt, Introduction to Functional Analysis, Oxford Graduate Texts in Mathematics, 2, The Clarendon Press, Oxford University Press, New York, 1997.

[MiWo] A. Miralles, E. Wolf, Hypercyclic composition operators on $H_{v}^{0}$-spaces, Math. Nachr. 286 (2013), no. 1, 34-41.

[Na] R. Narasimhan, Analysis on Real and Complex Manifolds, Advanced Studies in Pure Mathematics, Vol. 1, North-Holland Publishing Co., Amsterdam, 1968.

[Pr] A. Przestacki, Dynamical properties of weighted composition operators on the space of smooth functions, J. Math. Anal. Appl. 445 (2017), no. 1, 1097-1113.

[RaSc1] A. Rainer, G. Schindl, Composition in ultradifferentiable classes, Studia Math. 224 (2014), no. 2, 97-131.

[RaSc2] A. Rainer, G. Schindl, Equivalence of stability properties for ultradifferentiable function classes, Rev. R. Acad. Cienc. Exactas Fís. Nat. Ser. A Math. RACSAM 110 (2016), no. 1, 17-32.

[Ru] W. Rudin, Real and Complex Analysis, McGraw-Hill, Third Edition, 1987.

[Sh] J. Shapiro Composition Operators and Classical Function Theory, Universitext: Tracts in Mathematics. Springer-Verlag, New York, 1993.

[Tr] F. Trèves, Topological Vector Spaces, Distributions, and Kernels, Academic Press, New York, 1967. 
[Wa] S. Warner, The topology of compact convergence on continuous function spaces, Duke Math. J. 25 (1958), 265-282.

[We] J. Wengenroth, Derived Functors in Functional Analysis, Lecture Notes in Mathematics, 1810, Springer, Berlin, 2003.

[YoRe] B. Yousefi, H. Rezaei, Hypercyclic property of weighted composition operators, Proc. Amer. Math. Soc. 135 (2007), no. 10, 3263-3271.

[Za] S. Zając, Hypercyclicity of composition operators in Stein manifolds, Proc. Amer. Math. Soc. 144 (2016), no. 9, 3991-4000.

Faculty of Mathematics, Chemnitz Technical University, 09107 Chemnitz, Germany

E-mail address: thomas.kalmes@math.tu-chemnitz.de 